\title{
ON THE NATURE OF THE ENIGMATIC OBJECT IRAS 19312+1950: A RARE PHASE OF MASSIVE STAR FORMATION?*
}

\author{
M. A. Cordiner ${ }^{1,2}$, A. C. A. Boogert ${ }^{3}$, S. B. Charnley ${ }^{1}$, K. Justtanont ${ }^{4}$, N. L. J. Cox ${ }^{5,6}$, R. G. Smith ${ }^{7}$, A. G. G. M. Tielens ${ }^{8}$, \\ E. S. Wirström ${ }^{4}$, S. N. Milam ${ }^{1}$, And J. V. Keane ${ }^{9}$ \\ ${ }^{1}$ Astrochemistry Laboratory, NASA Goddard Space Flight Center, Code 691, 8800 Greenbelt Road, Greenbelt, MD 20771, USA; martin.cordiner@nasa.gov \\ ${ }^{2}$ Department of Physics, The Catholic University of America, Washington, DC 20064, USA \\ ${ }^{3}$ Universities Space Research Association, Stratospheric Observatory for Infrared Astronomy, \\ NASA Ames Research Center, MS 232-11, Moffett Field, CA 94035, USA \\ ${ }^{4}$ Department of Earth and Space Sciences, Chalmers University of Technology, Onsala Space Observatory, SE-439 92, Onsala, Sweden \\ ${ }^{5}$ Instituut voor Sterrenkunde, KU Leuven, Celestijnenlaan 200D, bus 2401, B-3001, Leuven, Belgium \\ ${ }^{6}$ Current address: Université de Toulouse, UPS-OMP, IRAP, F-31028 Toulouse, France \\ ${ }^{7}$ School of Physical, Environmental \& Mathematical Sciences, The University of New South Wales, \\ Australian Defence Force Academy, Canberra ACT 2600, Australia \\ ${ }^{8}$ Leiden Observatory, University of Leiden, P.O. Box 9513, NL-2300 RA Leiden, The Netherlands \\ ${ }_{9}$ Institute for Astronomy, University of Hawaii, Honolulu, HI 96822, USA \\ Received 2016 March 1; revised 2016 June 21; accepted 2016 June 30; published 2016 August 29
}

\begin{abstract}
IRAS $19312+1950$ is a peculiar object that has eluded firm characterization since its discovery, with combined maser properties similar to an evolved star and a young stellar object (YSO). To help determine its true nature, we obtained infrared spectra of IRAS $19312+1950$ in the range 5-550 $\mu \mathrm{m}$ using the Herschel and Spitzer space observatories. The Herschel PACS maps exhibit a compact, slightly asymmetric continuum source at $170 \mu \mathrm{m}$, indicative of a large, dusty circumstellar envelope. The far-IR CO emission line spectrum reveals two gas temperature components: $\approx 0.22 M_{\odot}$ of material at $280 \pm 18 \mathrm{~K}$, and $\approx 1.6 M_{\odot}$ of material at $157 \pm 3 \mathrm{~K}$. The O I $63 \mu \mathrm{m}$ line is detected on-source but no significant emission from atomic ions was found. The HIFI observations display shocked, high-velocity gas with outflow speeds up to $90 \mathrm{~km} \mathrm{~s}^{-1}$ along the line of sight. From Spitzer spectroscopy, we identify ice absorption bands due to $\mathrm{H}_{2} \mathrm{O}$ at $5.8 \mu \mathrm{m}$ and $\mathrm{CO}_{2}$ at $15 \mu \mathrm{m}$. The spectral energy distribution is consistent with a massive, luminous $\left(\sim 2 \times 10^{4} L_{\odot}\right)$ central source surrounded by a dense, warm circumstellar disk and envelope of total mass $\sim 500-700 M_{\odot}$, with large bipolar outflow cavities. The combination of distinctive far-IR spectral features suggest that IRAS 19312+1950 should be classified as an accreting, highmass YSO rather than an evolved star. In light of this reclassification, IRAS $19312+1950$ becomes only the fifth high-mass protostar known to exhibit $\mathrm{SiO}$ maser activity, and demonstrates that $18 \mathrm{~cm} \mathrm{OH}$ maser line ratios may not be reliable observational discriminators between evolved stars and YSOs.
\end{abstract}

Key words: ISM: molecules - masers - stars: AGB and post-AGB - stars: formation - stars: protostars - stars: winds, outflows

\section{INTRODUCTION}

IRAS $19312+1950$ is an infrared-bright object located in the Galactic plane at a distance of about $3.8 \mathrm{kpc}$ (Imai et al. 2011). Observations by 2MASS show an extended, horn-like IR nebulosity surrounding the central (point-like) source (Nakashima \& Deguchi 2000). A more recent near-IR (JHK) image from the UKIRT Infrared Deep Sky Survey is shown in Figure 1, in which a complex filamentary structure is evident, spanning a scale of $\sim 30^{\prime \prime}$.

Based on $\mathrm{SiO}$ and $\mathrm{H}_{2} \mathrm{O}$ maser detections, Nakashima \& Deguchi (2000) deduced that the central source of IRAS 19312 +1950 is most likely an oxygen-rich evolved star similar to $\mathrm{OH} 231.8+4.2$. Subsequent molecular line observations by Deguchi et al. (2004) identified abundant carbon-, nitrogen-, and oxygen-bearing molecules in the circumstellar envelope, many of which show a complex kinematical structure. The presence of a strong, narrow, bipolar emission component (about $1-2 \mathrm{~km} \mathrm{~s}^{-1}$ wide) superimposed on a broader component with FWHM $\sim 50 \mathrm{~km} \mathrm{~s}^{-1}$ led Deguchi et al. (2004) and Nakashima \& Deguchi (2005) to interpret the observed

\footnotetext{
* Herschel is an ESA space observatory with science instruments provided by European-led Principal Investigator consortia and with important participation from NASA.
}

emission as arising in the outflows from an AGB stellar atmosphere. However, several observational characteristics of IRAS $19312+1950$ are unusual for an AGB star, which justifies a closer look at the nature of this object: (1) the linear distribution of $\mathrm{H}_{2} \mathrm{O}$ maser spots and complex $\mathrm{OH}$ maser line profile (Nakashima et al. 2011); (2) the detection of common interstellar molecules, including $\mathrm{CH}_{3} \mathrm{OH}, \mathrm{N}_{2} \mathrm{H}^{+}$, and $\mathrm{HC}_{3} \mathrm{~N}$, which are not normally seen in O-rich AGB star envelopes; and (3) the association of IRAS $19312+1950$ with a massive, dense molecular cloud core G055.372+00.185 (Dunham et al. 2011).

Nakashima et al. (2011) identified the two most likely scenarios to explain the observations of IRAS 19312+1950. First, they considered the central object to be a massive $\left(\sim 10 M_{\odot}\right)$, O-rich AGB star with a fast bipolar outflow embedded in a chemically rich molecular cloud. Deguchi et al. (2004) noted that the association with a compact molecular cloud is highly unusual, but could be the result of a chance encounter (e.g., Kastner \& Myers 1994). On the other hand, the evidence could be indicative that IRAS 19312+1950 is an unusual, massive young stellar object (YSO), which would be consistent with some of the observed outflow characteristics as well as the high $\mathrm{CH}_{3} \mathrm{OH}$ and $\mathrm{HC}_{3} \mathrm{~N}$ abundances. A Class $\mathrm{I} \mathrm{CH}_{3} \mathrm{OH}$ maser was recently detected in IRAS $19312+1950$ by Nakashima et al. (2015). This class 


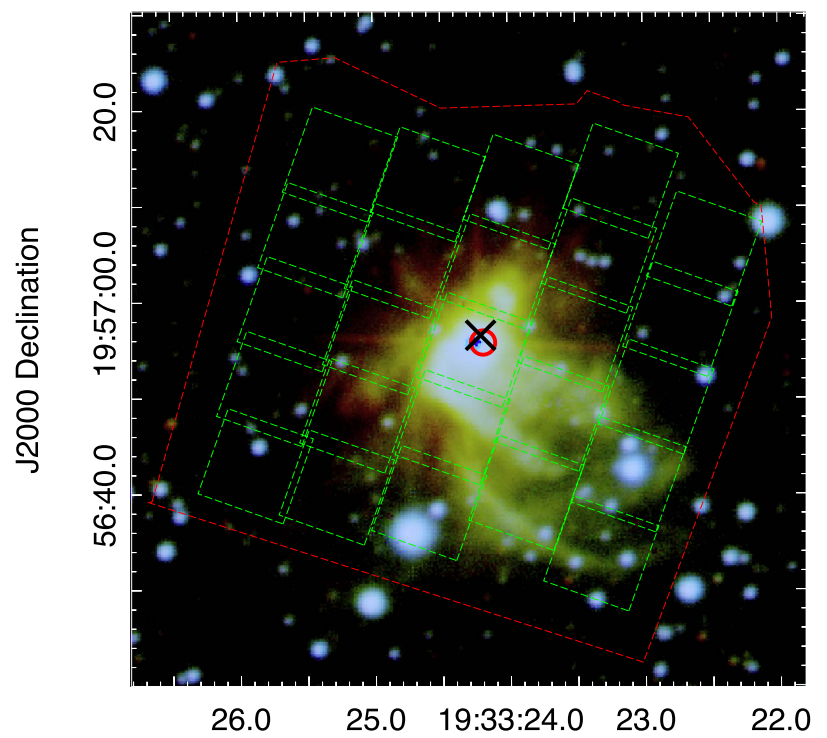

J2000 Right Ascension

Figure 1. UKIDSS $J$ (blue), $H$ (green), and $K$ (red) image of IRAS 19312 +1950 with $5 \times 5$ PACS spaxel footprint overlaid (green dashed boxes). Brightness histograms have been stretched to emphasize the IR nebulosity. Each PACS spaxel has dimensions of 9 !. $4 \times 9$ ". 4 . The image is centered on the location of the 2MASS point source. The position of the peak $170 \mu \mathrm{m}$ continuum flux is shown with a black " $\times$." The red circle indicates the CO farIR emission peak. The red dashed outline indicates the $\left(\approx 54^{\prime \prime} \times 54^{\prime \prime}\right)$ region covered by the PACS raster maps. For details of the UKIDSS project, see Lawrence et al. (2007). UKIDSS uses the UKIRT Wide Field Camera (WFCAM; Casali et al. 2007); the photometric system and calibration were described by Hewett et al. (2006) and Hodgkin et al. (2009), respectively.

of maser has not previously been seen in evolved stars, but is common in regions of high-mass star formation as a tracer of molecular outflows (e.g., Cyganowski et al. 2009). Strongly variable red- and blueshifted $43 \mathrm{GHz} \mathrm{SiO}$ maser peaks were observed toward IRAS $19312+1950$ by Scott (2002) and Nakashima et al. (2011), with a velocity separation of about $30-36 \mathrm{~km} \mathrm{~s}^{-1}$. Scott (2002) speculated that these masers could arise in the approaching and receding sides of a rotating disk about a YSO, similar to the $\mathrm{SiO}$ masers seen in Orion KL. SiO masers, however, are extremely rare in YSOs, having been seen in only four massive star-forming regions to date: Orion KL, Sgr B2, W51 (Zapata et al. 2009), and recently in Galactic Center Cloud C, G0.38+0.04 (Ginsburg et al. 2015). By contrast, $\mathrm{SiO}$ masers have been observed in thousands of AGB stars. Furthermore, the strong $1612 \mathrm{MHz} \mathrm{OH}$ maser line of IRAS $19312+1950$ (relative to the 1665 and $1667 \mathrm{MHz}$ lines) is a typical characteristic of evolved stars (Caswell 1998; Nakashima et al. 2011), so that if confirmed as a YSO, the combined maser properties of IRAS $19312+1950$ would be remarkable.

The spectral energy distribution (SED) of IRAS 19312 +1950 was modeled by Murakawa et al. (2007), who assumed the central object to be a high mass-loss AGB star. At the time of that study, the SED was not well constrained in the far-IR. A severe mismatch between the model and observations at the longest wavelengths $(>20 \mu \mathrm{m})$ was dismissed as primarily the result of background flux contamination. However, the large far-infrared fluxes measured by IRAS indicate that the circumstellar envelope mass (as well as the intrinsic source luminosity and temperature) may have been underestimated. If confirmed, an SED that rises to a peak in the far-IR would be more reminiscent of a young, massive YSO embedded in a dense, accreting envelope.

To help determine the true nature of IRAS $19312+1950$, we have obtained mid- and far-IR spectroscopic observations to probe the properties of the environment surrounding this enigmatic, dust-enshrouded object. The Herschel Space Observatory (Pilbratt et al. 2010) HIFI (Heterodyne Instrument for the Far-Infrared; de Graauw et al. 2010) and PACS (Photodetector Array Camera \& Spectrometer; Poglitsch et al. 2010) instruments were used to observe emission from dust, carbon monoxide, water, and other species in the wavelength range 51-550 $\mu \mathrm{m}$. Spitzer Space Telescope spectra were also obtained, covering the range 5-35 $\mu \mathrm{m}$. The combined Herschel and Spitzer observations provide crucial new information on the SED and ice properties, complementing the previous IR data from ISO, 2MASS, Akari, the Wide-field Infrared Survey Explorer (WISE), and other telescopes. In addition, our high signal-to-noise, spectrally resolved HIFI CO and $\mathrm{H}_{2} \mathrm{O}$ line profiles provide new information on the nature of the outflow. The evidence provided by these observations strongly favor the identification of IRAS $19312+1950$ as an emdedded, high-mass YSO.

\section{OBSERVATIONS AND DATA REDUCTION}

\subsection{Herschel Observations}

Herschel observations of IRAS $19312+1950$ were obtained between 2012 May and October as part of Cycle 2 Open Time Programme OT2_mcordine_2. Basic observational parameters and instrument settings are given in Table 1. Four PACS pointings in a $2 \times 2$-pointing raster pattern were obtained around the central source position (R.A. 19:33:24.29, decl. 19:56:55.0 (J2000)), each employing one repetition of the B2A (short) and B2B (long) spectral scan modes to cover the complete wavelength range 51-220 $\mu \mathrm{m}$. Observations were obtained in chop/nod mode, using a $6^{\prime}$ throw on either side of the source (at an angle $30^{\circ}$ clockwise from celestial north). The PACS integral-field unit (IFU) consists of a $5 \times 5$ array of 9." 4-square spaxels and the spacing between map pointings was 4". 5 in R.A. and decl. This ensured full sampling of the spatial point-spread function (PSF), which has a minimum FWHM of about $9^{\prime \prime}$ at $50 \mu \mathrm{m}$ (rising to about $13^{\prime \prime}$ at $180 \mu \mathrm{m}$ ). The relative locations and shapes of the PACS IFU spaxel array elements are shown overlaid in green over the UKIDSS ( $J H K$ ) image of IRAS $19312+1950$ in Figure 1.

The PACS range-scan data were reduced using HIPE pipeline version 11.0 (Ott 2010), which included standard flat-fielding, deglitching, wavelength and flux calibration routines, as well as projecting of the data into threedimensional (3D) raster maps with a plate scale of $3^{\prime \prime} /$ pixel. Data at wavelengths $<55 \mu \mathrm{m}$ in the B2A blue spectra, $>98 \mu \mathrm{m}$ in the B2B blue spectra, and $>189 \mu \mathrm{m}$ were excluded from subsequent analysis due to contamination by reflected light inside the PACS spectrometer. Absolute Flux calibration is expected to be accurate to within about $10 \%$.

PACS spectra were extracted by summation over a $54^{\prime \prime}$ diameter circular aperture at each wavelength. The majority of the flux from IRAS $19312+1950$ (see, e.g., Figure 3) is contained in this region. IRAS $19312+1950$ appears to be the only IR-bright object within the mapped region (also confirmed by 2 MASS and UKIDSS imaging), so contamination from other sources is expected to be negligible. To measure the 
Table 1

Herschel Observations of IRAS $19312+1950$

\begin{tabular}{|c|c|c|c|c|c|c|c|}
\hline OBSID & Date & R.A. & decl. & Instrument & Mode & Setting & Duration (s) \\
\hline 1342245229 & 2012 May 01 & $19: 33: 24.3$ & $+19: 56: 55$ & PACS & Range Scan & $\mathrm{B} 2 \mathrm{~B}$ & 9134 \\
\hline 1342245230 & 2012 May 01 & $19: 33: 24.3$ & $+19: 56: 55$ & PACS & Range Scan & B2A & 4616 \\
\hline 1342245377 & 2012 May 04 & $19: 33: 24.3$ & $+19: 56: 55$ & HIFI & WBS DBS & $1102+1114$ & 3286 \\
\hline 1342245378 & 2012 Мay 04 & $19: 33: 24.3$ & $+19: 56: 55$ & HIFI & WBS DBS & $1097+1109$ & 2028 \\
\hline 1342245380 & 2012 May 04 & $19: 33: 24.3$ & $+19: 56: 55$ & HIFI & WBS DBS & $546+558$ & 758 \\
\hline 1342252110 & 2012 Oct 02 & $19: 33: 24.3$ & $+19: 56: 55$ & HIFI & WBS DBS & $647+659$ & 2448 \\
\hline 1342252111 & 2012 Oct 02 & $19: 33: 24.3$ & $+19: 56: 55$ & HIFI & WBS DBS & $679+691$ & 1548 \\
\hline 1342255788 & 2012 Nov 22 & $19: 33: 24.3$ & $+19: 56: 55$ & HIFI & WBS DBS & $576+588$ & 894 \\
\hline 1342255789 & 2012 Nov 22 & $19: 33: 24.3$ & $+19: 56: 55$ & HIFI & WBS DBS & $608+621$ & 739 \\
\hline
\end{tabular}

Note. HIFI settings indicate the central frequencies (in GHz) of the lower and upper receiver sidebands, respectively (each sideband is $4 \mathrm{GHz}$ wide).

Table 2

HIFI Integrated Line Fluxes

\begin{tabular}{|c|c|c|c|c|c|c|c|c|}
\hline Species & Transition & $\eta_{\mathrm{MB}}$ & $\begin{array}{c}\mathrm{HPBW} \\
\left({ }^{\prime \prime}\right)\end{array}$ & $\begin{array}{c}\lambda \\
(\mu \mathrm{m})\end{array}$ & $\begin{array}{c}\nu \\
(\mathrm{GHz})\end{array}$ & $\begin{array}{c}E_{u} \\
(\mathrm{~K})\end{array}$ & $\underset{(\mathrm{Jy} G \mathrm{GH})}{S_{\nu} d \nu}$ & $\begin{array}{c}\int T_{\mathrm{MB}} d v \\
\left(\mathrm{~K} \mathrm{~km} \mathrm{~s}^{-1}\right)\end{array}$ \\
\hline $\mathrm{CO}$ & $5-4$ & 0.62 & 37 & 520.231 & 576.267 & 82.98 & $79.33(0.05)$ & $126.27(0.07)$ \\
\hline $\mathrm{CO}$ & $6-5$ & 0.65 & 31 & 433.556 & 691.473 & 116.2 & $115.79(0.05)$ & $153.58(0.07)$ \\
\hline${ }^{13} \mathrm{CO}$ & $6-5$ & 0.65 & 32 & 453.498 & 661.067 & 111.1 & $9.16(0.03)$ & $12.72(0.06)$ \\
\hline${ }^{13} \mathrm{CO}$ & $10-9$ & 0.64 & 19 & 272.205 & 1101.35 & 290.8 & $4.50(0.06)$ & $3.75(0.06)$ \\
\hline $\mathrm{C}^{18} \mathrm{O}$ & $6-5$ & 0.65 & 32 & 455.229 & 658.553 & 110.6 & $1.05(0.02)$ & $1.47(0.02)$ \\
\hline $\mathrm{C}^{18} \mathrm{O}$ & $10-9$ & 0.64 & 19 & 273.243 & 1097.16 & 289.7 & $0.32(0.03)$ & $0.27(0.03)$ \\
\hline $\mathrm{p}-\mathrm{H}_{2} \mathrm{O}$ & $1_{11}-0_{00}$ & 0.64 & 19 & 269.272 & 1113.34 & 53.43 & $21.77(0.13)$ & $17.93(0.10)$ \\
\hline $\mathrm{o}-\mathrm{H}_{2} \mathrm{O}$ & $1_{10}-1_{01}$ & 0.62 & 38 & 538.289 & 556.936 & 60.97 & $4.96(0.04)$ & $8.18(0.07)$ \\
\hline $\mathrm{o}-\mathrm{H}_{2} \mathrm{O}$ & $3_{12}-3_{03}$ & 0.64 & 19 & 273.193 & 1097.36 & 249.4 & $16.03(0.10)$ & $13.39(0.08)$ \\
\hline $\mathrm{o}-\mathrm{H}_{2}{ }^{18} \mathrm{O}$ & $1_{10}-1_{01}$ & 0.62 & 39 & 547.390 & 547.676 & 60.46 & $0.20(0.02)$ & $0.33(0.04)$ \\
\hline $\mathrm{HCN}$ & $7-6$ & 0.62 & 34 & 483.299 & 620.304 & 119.1 & $1.15(0.02)$ & $1.71(0.04)$ \\
\hline CS & $12-11$ & 0.62 & 36 & 510.184 & 587.616 & 183.4 & $0.09(0.01)$ & $0.13(0.01)$ \\
\hline $\mathrm{SiO}$ & $14-13$ & 0.62 & 35 & 493.398 & 607.608 & 218.8 & $0.11(0.01)$ & $0.17(0.02)$ \\
\hline
\end{tabular}

Note. Statistical $\pm 1 \sigma$ integrated line brightness/flux errors are given in parentheses.

spectral line fluxes, wavelengths were converted to frequencies, then baseline-subtracted using low-order polynomials. Observed line widths of $325 \mathrm{~km} \mathrm{~s}^{-1}$ at $108 \mu \mathrm{m}$ and $212 \mathrm{~km} \mathrm{~s}^{-1}$ at $174 \mu \mathrm{m}$ match the PACS instrument specifications for the spectroscopic resolving power, indicating that these lines were not resolved.

The HIFI instrument was used to obtain single-pointing, dual-polarization, double sideband observations of a selection of transitions from $\mathrm{CO}$ and $\mathrm{H}_{2} \mathrm{O}$ toward the center of IRAS 19312+1950. These were carried out using the WideBand Spectrometer (WBS), and the High Resolution Spectrometer (HRS) simultaneously, with spectral resolutions of 1.1 MHz and $0.25 \mathrm{MHz}$, respectively. Dual beam-switching (DBS) mode was used to remove the instrumental background signal. The detected lines and their respective wavelengths and frequencies are given in Table 2.

The HIFI spectra were reduced using the HIPE pipeline (version 9.0) then baseline-subtracted using low-order polynomials fitted to the continuum on either side of the lines of interest. Differences in intensity between the two polarizations were negligible, and so these were averaged to improve the signal-to-noise ratio. Spectral line antenna temperatures were corrected to the main beam $\left(T_{\mathrm{MB}}\right)$ scale using the beam efficiencies in Table 2 and a telescope forward efficiency factor of 0.96. Average continuum fluxes from the two sidebands were measured (before baseline subtraction) in regions free from any spectral lines.

\subsection{Spitzer Observations}

Spectra of IRAS $19312+1950$ were obtained with the Infrared Spectrograph (IRS) of the Spitzer Space Telescope on 2006 October 19 during IRS campaign 1070 (IRSX007500), as part of Guaranteed Time Observer program 93 (principal investigator D. Cruikshank). The IRS modules Short-Low (SL; 5.2-14.5 $\mu \mathrm{m}$ at $R=\lambda / \Delta \lambda \sim 100$ ), Long-Low (LL; 14-38 $\mu \mathrm{m}$ at $R \sim 100$ ), and Short-High (SH; 9.9-19.6 $\mu \mathrm{m}$ at $R \sim 600$ ) were all observed in staring mode at the default two nodding positions along the slit and also at two positions on the sky, one centered on IRAS $19312+1950$, and one on a position offset $10^{\prime \prime}$ to the east and $10^{\prime \prime}$ to the north. In all of the modules, the emission in the offset position is negligible compared to the main target, and is therefore not used in the analysis. High pointing accuracy was guaranteed by using the optical PCRS peak up mode on a nearby star. Ramp times of 14 and $6 \mathrm{~s}$ were used for the low (SL and LL) and high (SH) resolution modules, respectively. SL was observed for 5 cycles, and LL and $\mathrm{SH}$ for 3 cycles.

The SL spectra were extracted and calibrated from the twodimensional Basic Calibrated Data (BCD) produced by the standard Spitzer pipeline (version S18.18.0), using the same method and routines discussed in Boogert et al. (2011). Uncertainties $(1 \sigma)$ for each spectral point were calculated using the "func" frames provided by the Spitzer pipeline. One section of the SL1 sub-module (11.0-13.0 $\mu \mathrm{m})$ suffers from saturation, recognizable by "NaN" values in the BCD images. The other 
spectral regions in SL do not contain saturation flags and are scientifically valid. All SL data points above $10.0 \mu \mathrm{m}$ were removed, however, as this region is covered by the SH module at higher spectral resolution. It was verified that overlapping unsaturated SL and SH data points are in good agreement. For the SH module, the one-dimensional spectra in the "tune" tables produced by the Spitzer pipeline were used. All data points with non-zero flag values were excluded and the spectra on the two nodding positions were averaged. This results in a nearly continuous $9.9-19.6 \mu \mathrm{m}$ spectrum not affected by saturation. The LL spectra, however, are highly saturated in the peak of the PSF and a special extraction was performed to mitigate the effects. Using the Spitzer IRS Custom Extraction (SPICE) software package, spectra were extracted along 3 and 6 pixel-wide rectangles. The resulting spectra were subtracted from each other, resulting in spectra tracing the regions 1.5-3 pixels $(7 ! \cdot 7-15$ !' 3$)$ on either side of the source. Such nonstandard extraction requires a dedicated spectral response function. The standard star for the IRS campaign (HR 6606) is weak in the PSF wings, however, and a new response function could not be derived. Following the IRS manual, 10\% uncertainties were assigned to the LL flux values thus derived.

The combined Spitzer spectrum was produced by multiplying the $\mathrm{SH}$ fluxes by a factor of 0.96 to match SL. The portion of LL overlapping with $\mathrm{SH}(14.0-19.6 \mu \mathrm{m})$ was removed, and the longer wavelengths were multiplied to match $\mathrm{SH}$. Although no emission was detected $10^{\prime \prime}$ north and east of the source, due to the special extraction method discussed above, it should be kept in mind that the LL spectrum could be contaminated by emission at a distances $7 ! 7-15 ! 3$ along the slit.

\section{RESULTS}

\subsection{PACS Range Scans}

PACS spectra in the range 54-189 $\mu \mathrm{m}$ are shown in Figure 2. A plethora of emission lines is present atop the strong (thermal) dust continuum. Most of the lines are due to $\mathrm{H}_{2} \mathrm{O}$ and $\mathrm{CO}$; transitions, wavelengths, and integrated line fluxes for these species are given in Tables 3 and 4. Integrated line fluxes are in units of $\mathrm{Jy} \mathrm{GHz}$, with $1 \sigma$ statistical errors derived from the rms noise in the nearby continuum.

Emission lines from O I (at $63 \mu \mathrm{m}$ and $146 \mu \mathrm{m}$ ), C II $(158 \mu \mathrm{m})$, and an $\mathrm{OH}$ doublet $(163 \mu \mathrm{m})$ are clearly visible, as well as absorption lines of $\mathrm{OH}(119 \mu \mathrm{m})$ and $\mathrm{CH}(149 \mu \mathrm{m})$. Broad structures in the two shorter-wavelength regions are found to be highly spatially variable across the small region mapped by PACS, and are likely to be a result of instrumental artifacts. All of the lines visible in Figure 2 have been identified. We searched for additional lines from species including $\mathrm{SiO}, \mathrm{HCN}$, and $\mathrm{NH}_{3}$ (and $\mathrm{CH}_{3} \mathrm{OH}$ ), which have previously been seen in the envelopes of oxygen-rich (and carbon-rich) AGB stars using PACS (Decin et al. 2010; Royer et al. 2010), but no emission from these species was detected. No evidence was found for $\mathrm{N}$ II or $\mathrm{O}$ III emission, which are common tracers of ultra-compact $\mathrm{HII} /$ massive star-forming regions (e.g., van Loon et al. 2010). The $69 \mu \mathrm{m}$ forsterite band (seen in evolved stellar envelopes) was also not detected.

\section{2. $\mathrm{CO}$ and $\mathrm{H}_{2} \mathrm{O}$ Rotational Diagrams}

Following the formalism described by Justtanont et al. (2000), rotational diagrams were generated for the $\mathrm{CO}$ and $\mathrm{H}_{2} \mathrm{O}$ lines observed with PACS. Neglecting optical depth and background radiation, the spectrally integrated line flux $(F)$ for a transition at frequency $\nu$ is related to the number of molecules in the upper energy state $\left(N_{u}\right)$ and the source distance $(d)$ by

$$
F=\int S_{\nu} d \nu=N_{u} \frac{A h \nu}{4 \pi d^{2}},
$$

where $A$ is the Einstein spontaneous decay rate. Therefore, in LTE at a temperature $T$,

$$
F=N \frac{e^{-E_{u} / k T}}{Q(T)} \frac{A h \nu g_{u}}{4 \pi d^{2}},
$$

where $N$ is the total number of molecules, $E_{u}$ is the upper-state energy, $g_{u}$ is the degeneracy, and $Q(T)$ is the partition function. Figure 4 shows the plots of $\ln \left(4 \pi F / A h \nu g_{u}\right)$ versus $E_{u}$ for $\mathrm{CO}$ and $\mathrm{H}_{2} \mathrm{O}$. The gradients of these diagrams are equal to $-1 / T$ and the intercepts give $\ln \left(N / Q(T) d^{2}\right)$. Adopting a distance of $3.8 \mathrm{kpc}$, linear least-squares fits (allowing for errors) result in the following parameters: $T=197.7 \pm 2.1 \quad \mathrm{~K}$, $N=(3.3 \pm 0.2) \times 10^{52}$ for $\mathrm{CO}$, and $T=80.5 \pm 1.4 \mathrm{~K}$, $N=(1.9 \pm 0.2) \times 10^{49}$ for $\mathrm{H}_{2} \mathrm{O}$. An equilibrium $\mathrm{H}_{2} \mathrm{O}$ orthoto-para ratio of three has been assumed.

From Figure 4, the $\mathrm{CO}$ rotational diagram is clearly comprised of two distinct temperature regimes, which indicates the presence of two gas components at different temperatures. These components were fitted separately, excluding the outlier point at $E_{u}=945 \mathrm{~K}$ that could be due to non-thermal excitation or blending with an unidentified emission line. The higher-temperature component has $T=280 \pm 18 \mathrm{~K}$, $N=8.4_{-2.3}^{+3.3} \times 10^{51}$, while the lower-temperature component has $T=157 \pm 3 \mathrm{~K}, N=6.3_{-0.6}^{+0.7} \times 10^{52}$. Assuming a $\mathrm{CO} / \mathrm{H}_{2}$ ratio of $10^{-4}$ and a mean mass per particle of $2.4 \mathrm{amu}$, the mass associated with the warmer component is $0.22 M_{\odot}$, and with the cooler component is $1.6 M_{\odot}$.

Rotational diagrams for evolved stellar atmospheres typically exhibit a relatively uniform slope, indicative of a single gas temperature component (e.g., Justtanont et al. 2000; Danilovich et al. 2014; Khouri et al. 2014). This is consistent with a single dominant heating source in the circumstellar envelope due to infrared irradiation by the dust-enshrouded central star. By contrast, YSOs tend to have a more complex heating environment, often resulting in multiple components with different temperatures (e.g., Fuente et al. 2005; Bruderer et al. 2009; Manoj et al. 2013; Karska et al. 2014; Lee et al. 2015). Three main mechanisms for the excitation of protostellar $\mathrm{CO}$ and $\mathrm{H}_{2} \mathrm{O}$ were considered by van Kempen et al. (2010) and Visser et al. (2012): (1) passive (IR) radiative heating of the envelope, (2) heating by UV radiation from the protostar, and (3) shock heating due to the impact of the fast bipolar outflow. They determined that shock heating of envelope cavity walls by the protostellar outflow (to temperatures up to $\gtrsim 1000 \mathrm{~K}$ ) is the main heating mechanism for far-IR $\mathrm{H}_{2} \mathrm{O}$ emission. This shock heating also results in the appearance of highly excited CO lines with $J_{u}>25$. The fact that our observed $\mathrm{CO}$ line fluxes (Table 3) fall rapidly with increasing $J$, with no detection of lines with $J_{u}>24$, indicates a lack of emission from shocked gas along the outflow cavity walls, so that strongly sub-thermal shock excitation or radiative heating by the (proto-)star is a likely explanation for the $\mathrm{CO}$ line excitation observed in IRAS $19312+1950$. The two CO temperature regimes found in IRAS $19312+1950$ are likely 

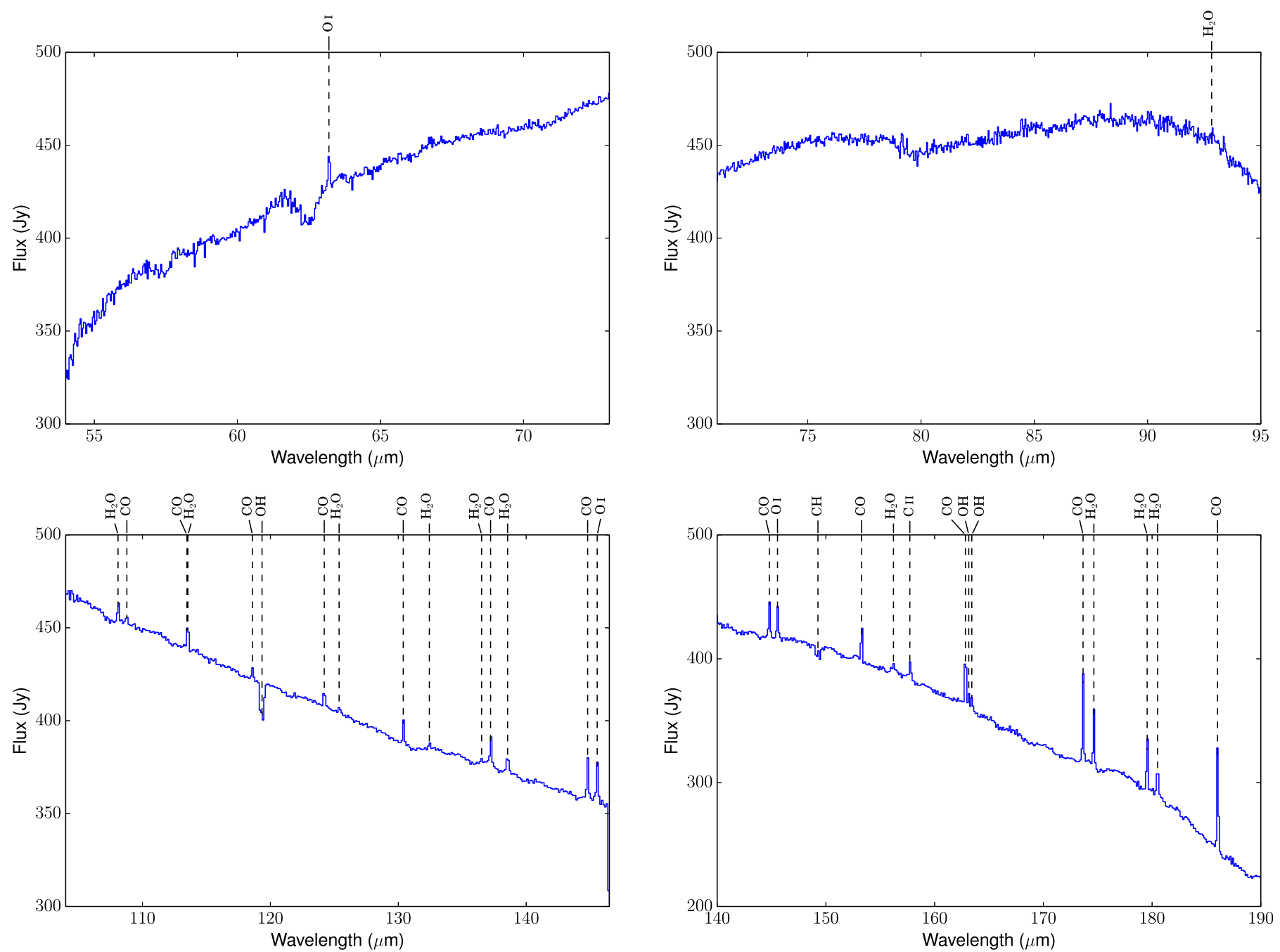

Figure 2. PACS range scans integrated over the a 54"-diameter circular aperture centered on IRAS 19312+1950. Assigned emission and absorption lines are marked. Apparent absorption features around 62.5 and $80 \mu \mathrm{m}$ are probably instrumental artifacts.

Table 3

PACS CO Integrated Line Fluxes

\begin{tabular}{lccc}
\hline \hline Transition & $\lambda(\mu \mathrm{m})$ & $E_{u}(\mathrm{~K})$ & $\int S_{\nu} d \nu(\mathrm{Jy} \mathrm{GHz})$ \\
\hline $14-13$ & 185.999 & 580.5 & $133.6(2.8)$ \\
$15-14$ & 173.631 & 663.4 & $112.7(1.6)$ \\
$16-15$ & 162.812 & 751.8 & $89.0(2.4)$ \\
$17-16$ & 153.266 & 845.6 & $63.5(1.7)$ \\
$18-17$ & 144.784 & 945.0 & $78.4(3.0)$ \\
$19-18$ & 137.196 & 1050 & $42.5(2.3)$ \\
$20-19$ & 130.369 & 1160 & $36.6(1.6)$ \\
$21-20$ & 124.193 & 1276 & $30.4(2.4)$ \\
$22-21$ & 118.581 & 1397 & $24.8(1.9)$ \\
$23-22$ & 113.457 & 1524 & $<69.1$ \\
$24-23$ & 108.763 & 1657 & $15.4(3.4)$ \\
\hline
\end{tabular}

Note. Statistical $1 \sigma$ errors are given in parentheses. The $J=23-22$ flux is an upper limit due to blending with the $\mathrm{H}_{2} \mathrm{O} \quad 4_{14}-3_{03}$ transition. 1 Jy GHz $=10^{-17} \mathrm{~W} \mathrm{~m}^{-2}$.

indicative of two distinct physical components in the source, perhaps including (but not limited to) a circumstellar disk and warm envelope or an outflow cavity wall component.

The CO rotational diagram is similar to those derived by Karska et al. (2014), who obtained best-fitting temperatures
Table 4

PACS $\mathrm{H}_{2} \mathrm{O}$ Integrated Line Fluxes

\begin{tabular}{lccc}
\hline \hline Transition & $\lambda(\mu \mathrm{m})$ & $E_{u}(\mathrm{~K})$ & $\int S_{\nu} d \nu(\mathrm{Jy} \mathrm{GHz})$ \\
\hline $6_{43}-6_{34}$ & 92.811 & 1089 & $<12.5$ \\
$2_{21}-1_{10}$ & 108.073 & 194.1 & $50.0(4.8)$ \\
$4_{14}-3_{03}$ & 113.537 & 323.5 & $<69.1$ \\
$4_{04}-3_{13}$ & 125.354 & 319.5 & $10.8(1.8)$ \\
$4_{23}-4_{14}$ & 132.408 & 432.2 & $11.8(1.8)$ \\
$3_{30}-3_{21}$ & 136.496 & 410.7 & $3.8(1.8)$ \\
$3_{13}-2_{02}$ & 138.528 & 204.7 & $32.1(2.3)$ \\
$3_{22}-3_{13}$ & 156.194 & 296.8 & $13.0(1.8)$ \\
$5_{32}-5_{23}$ & 160.510 & 732.1 & $<2.8$ \\
$7_{34}-7_{25}$ & 166.815 & 1212 & $<2.7$ \\
$3_{03}-2_{12}$ & 174.626 & 196.8 & $85.0(2.3)$ \\
$2_{12}-1_{01}$ & 179.526 & 114.4 & $61.0(2.7)$ \\
$2_{21}-2_{12}$ & 180.488 & 194.1 & $46.7(3.5)$ \\
\hline
\end{tabular}

Note. Statistical $1 \sigma$ errors are given in parentheses. Upper limits are $3 \sigma \Delta \nu$, except for $\mathrm{H}_{2} \mathrm{O}$ 414-303, which is blended with $\mathrm{CO}$ 23-22. 1 $\mathrm{Jy} \mathrm{GHz}=10^{-17} \mathrm{~W} \mathrm{~m}^{-2}$.

$\sim 300 \mathrm{~K}$ toward 10 high-mass protostars. Karska et al. (2014) also did not detect the hot, high-J CO component previously observed in low-mass sources, but nevertheless deduced that 

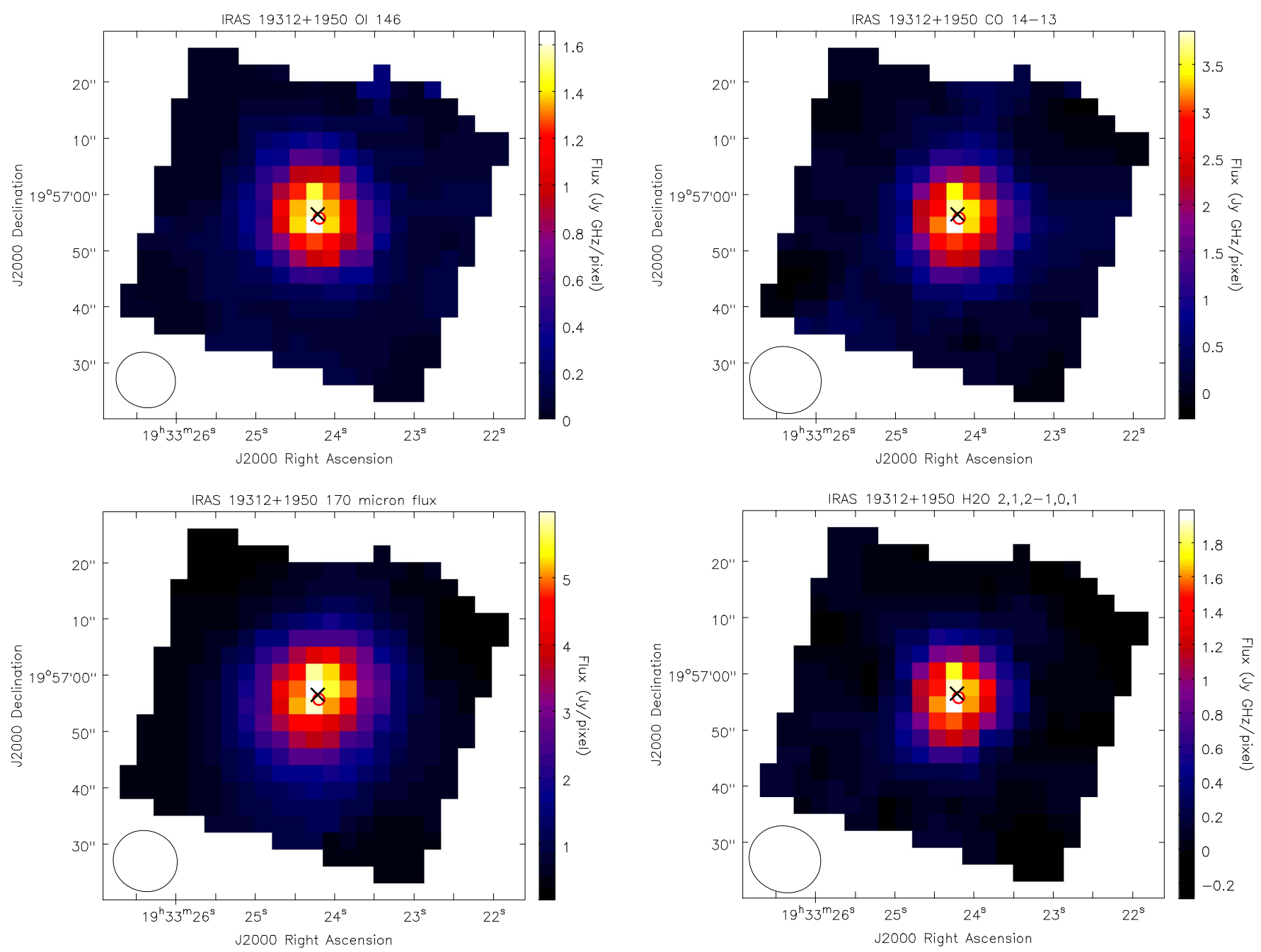

Figure 3. Top left: PACS O I $146 \mu \mathrm{m}$ flux raster map. Bottom left: PACS $170 \mu \mathrm{m}$ continuum flux raster map. Top right: PACS CO 14-13 flux raster map. Bottom right: PACS $\mathrm{H}_{2} \mathrm{O} 2_{1,2}-1_{0,1}$ flux raster map. All line flux maps are continuum-subtracted. The FWHMs of elliptical Gaussian fits to the PACS PSF are shown lower left in each panel. Black crosses show the position of the peak O I and $170 \mu$ m continuum flux. Red circles indicate the $\mathrm{CO}\left(\mathrm{and} \mathrm{H}_{2} \mathrm{O}\right.$ ) far-IR emission peak position. Peak positions were identified using two-dimensional Gaussian fits.
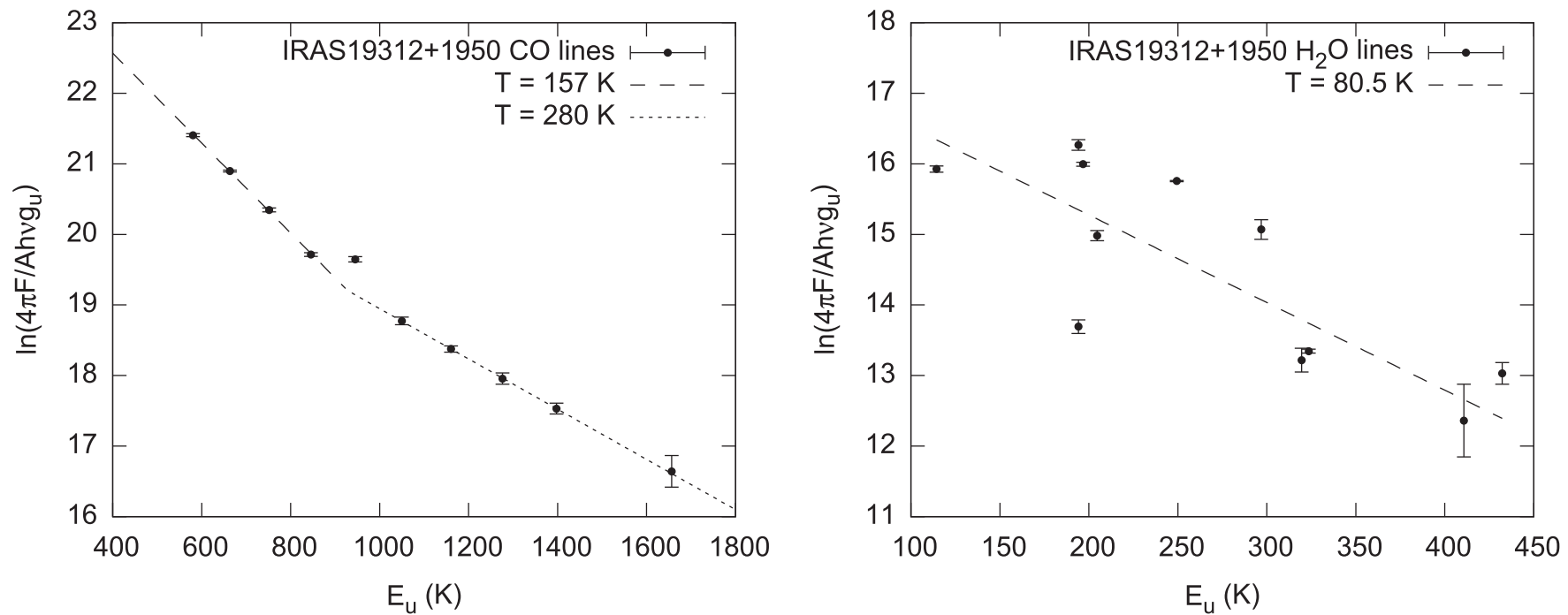

Figure 4. Rotational excitation diagram for $\mathrm{CO}$ (left) and $\mathrm{H}_{2} \mathrm{O}$ (right) derived from PACS range-scan integrated line fluxes. 
the observed warm PACS CO emission from massive YSOs probably originates in the heated outflow cavity walls.

The approximate impact of optical depth on these results may be examined by considering the $\mathrm{CO}$ and $\mathrm{H}_{2} \mathrm{O}$ column densities. Assuming the molecules are evenly distributed across the $\approx 10^{\prime \prime}$ PACS beam surrounding the source (corresponding to a radius of $19,000 \mathrm{au}$ at $3.8 \mathrm{kpc}$ ), the column densities for the warm $\mathrm{CO}$ and $\mathrm{H}_{2} \mathrm{O}$ detected by PACS are $(4.1 \pm 0.3) \times 10^{16}$ $\mathrm{cm}^{-2}$ and $(2.4 \pm 0.3) \times 10^{13} \mathrm{~cm}^{-2}$, respectively. Based on a lower limit to the line FWHM of $3 \mathrm{~km} \mathrm{~s}^{-1}$, derived from a Gaussian fit to the HIFI $\mathrm{C}^{18} \mathrm{O} 6-5$ line, the $\mathrm{CO}$ lines observed with PACS are all found to have optical depths $\tau<0.5$, and those with $J_{u}>17$ have $\tau<0.1$. The low-temperature component traced by the lower- $J$ lines may therefore suffer from some self absorption, whereas the higher-temperature component is likely optically thin. Several of the $\mathrm{H}_{2} \mathrm{O}$ line fluxes, on the other hand, may suffer more severely from opacity (due to the relatively lower $E_{u}$ values, which more closely match the apparent excitation temperature of the gas). The $2_{21}-1_{10}$ and $2_{12}-1_{01}$ lines, for example (at $E_{u}=194 \mathrm{~K}$ ), have $\tau \approx 0.7$, and most of the other lines have $\tau \gtrsim 0.1$. Self absorption may thus at least partly explain the scatter observed in the $\mathrm{H}_{2} \mathrm{O}$ rotational diagram. In addition to optical depth effects, $\mathrm{H}_{2} \mathrm{O}$ excitation modeling by Visser et al. (2012) showed that the PACS $\mathrm{H}_{2} \mathrm{O}$ rotational excitation by outflow shock heating results in non-LTE level populations and consequent scatter in the rotational diagram.

\subsection{O I lines}

Integrated fluxes for the $\mathrm{O} \mathrm{I}^{3} \mathrm{P}_{1}-{ }^{3} \mathrm{P}_{2}(63 \mu \mathrm{m})$ and ${ }^{3} \mathrm{P}_{0}-{ }^{3} \mathrm{P}_{1}$ $(146 \mu \mathrm{m})$ lines toward IRAS $19312+1950$ are $82 \pm 7 \mathrm{Jy} \mathrm{GHz}$ and $52 \pm 2 \mathrm{Jy} \mathrm{GHz}$, respectively, leading to a $63 / 146 \mu \mathrm{m}$ flux ratio of about 1.6. Emission from far-IR O I lines occurs in hot gas $(T \gtrsim 100 \mathrm{~K})$ where the fine-structure levels are excited by collisions with electrons and atomic/molecular hydrogen, and is typically seen in PDRs (see, for example, Hollenbach \& Tielens 1997) and YSOs (Liseau et al. 2006; van Kempen et al. 2010; Goicoechea et al. 2012), but may also be present in UV-irradiated outflows from evolved stars (Groenewegen et al. 2011). The low observed $63 / 146 \mu \mathrm{m}$ flux ratio is difficult to reproduce using single-component excitation models (Liseau et al. 2006), and is at variance with the values of $~ 20$ typically observed in YSOs using Herschel (e.g., Wampfler et al. 2010). Self absorption of the $63 \mu \mathrm{m}$ line in tenuous foreground gas was hypothesized by Liseau et al. (2006) as a possible explanation for such anomalously low ratios, which seems plausible given the likely presence of diffuse/translucent gas along the line of sight to IRAS $19312+1950$, which passes through the Galactic plane (see Section 3.5).

\subsection{PACS Maps}

Spectrally integrated PACS raster maps for several emission lines of interest, plus the $170 \mu \mathrm{m}$ continuum emission, are shown in Figure 3. These maps exhibit a striking similarity, consistent with the presence of a dense, compact, dust and gasrich envelope.

The total emitted flux at at $170 \mu \mathrm{m}$ is strong (330 Jy), and peaks at a position consistent with the near-IR source (Figure 1). This wavelength traces predominantly cold $(T \sim 10-20 \mathrm{~K})$ dust, and the close spatial correspondence indicates that the near-IR source is likely embedded near the center of a nearly spherical dusty envelope. The $170 \mu \mathrm{m}$ morphology is not well resolved but shows evidence for a slightly asymmetric, spatially extended structure, with a long axis FWHM of $23^{\prime \prime}$ aligned $35^{\circ}$ clockwise from north. The FWHM of the short axis is $20^{\prime \prime}$, which is still significantly extended compared with the $11^{\prime \prime}$ PACS PSF.

Examples of integrated $\mathrm{CO}$ and $\mathrm{H}_{2} \mathrm{O}$ line intensity maps are also presented in Figure 3, showing closely similar morphologies between these species, with emission strongly concentrated close to the central source. These maps also show evidence for elongation in approximately a $\mathrm{N}-\mathrm{S}$ direction, indicating the presence of an extended molecular envelope or perhaps an unresolved bipolar outflow structure.

Similar to the molecular gas and dust, the O I $146 \mu \mathrm{m}$ emission also peaks up strongly on the central position of IRAS $19312+1950$. Emission from C II at $158 \mu \mathrm{m}$ was detected in our PACS field in the vicinity of IRAS 19312 +1950 , with a relatively smooth distribution. However, a similar level of uniform C II emission was also detected in the off-source PACS nodding positions $6^{\prime}$ away. Foreground/ background Galactic C II emission from the diffuse ISM is a likely explanation for this emission, precluding the use of C II as a reliable tracer for ionized gas associated with IRAS $19312+1950$.

\subsection{Hi-Gal Imaging}

Figure 5 shows a composite image of PACS $70 \mu \mathrm{m}$ and $160 \mu \mathrm{m}$ emission in the region surrounding IRAS 19312 +1950 . This field was extracted from Herschel observations (OBSIDs 1342219812 and 1342219813), obtained as part of the Hi-Gal Galactic Plane Survey (Molinari et al. 2010; Traficante et al. 2011). The maps were reduced using the Scanamorphos software (Roussel 2013). Several evolved stars (AGB/PN) and (candidate) YSOs are located within the mapped region (Robitaille et al. 2008), indicating past and present star formation in field. Numerous other compact sources nearby await classification.

Emission in the 70 and $160 \mu \mathrm{m}$ wave bands traces predominantly cool and cold dust, and the complex structure across this region highlights the presence of numerous interstellar clouds, clumps, filaments and wind-blown bubbles at various distances along this line of sight through the Galactic mid-plane (at Galactic coordinates $l=55^{\circ} 4, \quad b=+0^{\circ} 2$ ). Despite its large distance $(\approx 3.8 \mathrm{kpc})$, the Hi-Gal imagery (in addition to 2MASS and UKIDSS) shows that IRAS 19312 +1950 is by far the brightest far-IR source in the region, and is host to a compact, luminous radiation source surrounded by a mass of cold dust that extends to a distance of at least $30^{\prime \prime}$ ( $\gtrsim 10^{5}$ au; see also Section 4).

\subsection{HIFI Spectra}

\subsubsection{HIFI Line Fluxes}

Baseline-subtracted, main beam efficiency-corrected HIFI WBS spectra are shown in Figure 6. Total integrated line fluxes are given in Table 2.

Due to the low intrinsic abundances of the minor isotopologues ${ }^{13} \mathrm{CO}$ and $\mathrm{C}^{18} \mathrm{O}$, the emission from these species should be quite optically thin. Using the ratio of intensities of the $J=10-9$ and 6-5 lines (employing a two-point rotational diagram, based on the method of Cummins et al. 1986), the excitation temperatures and column densities of these species 


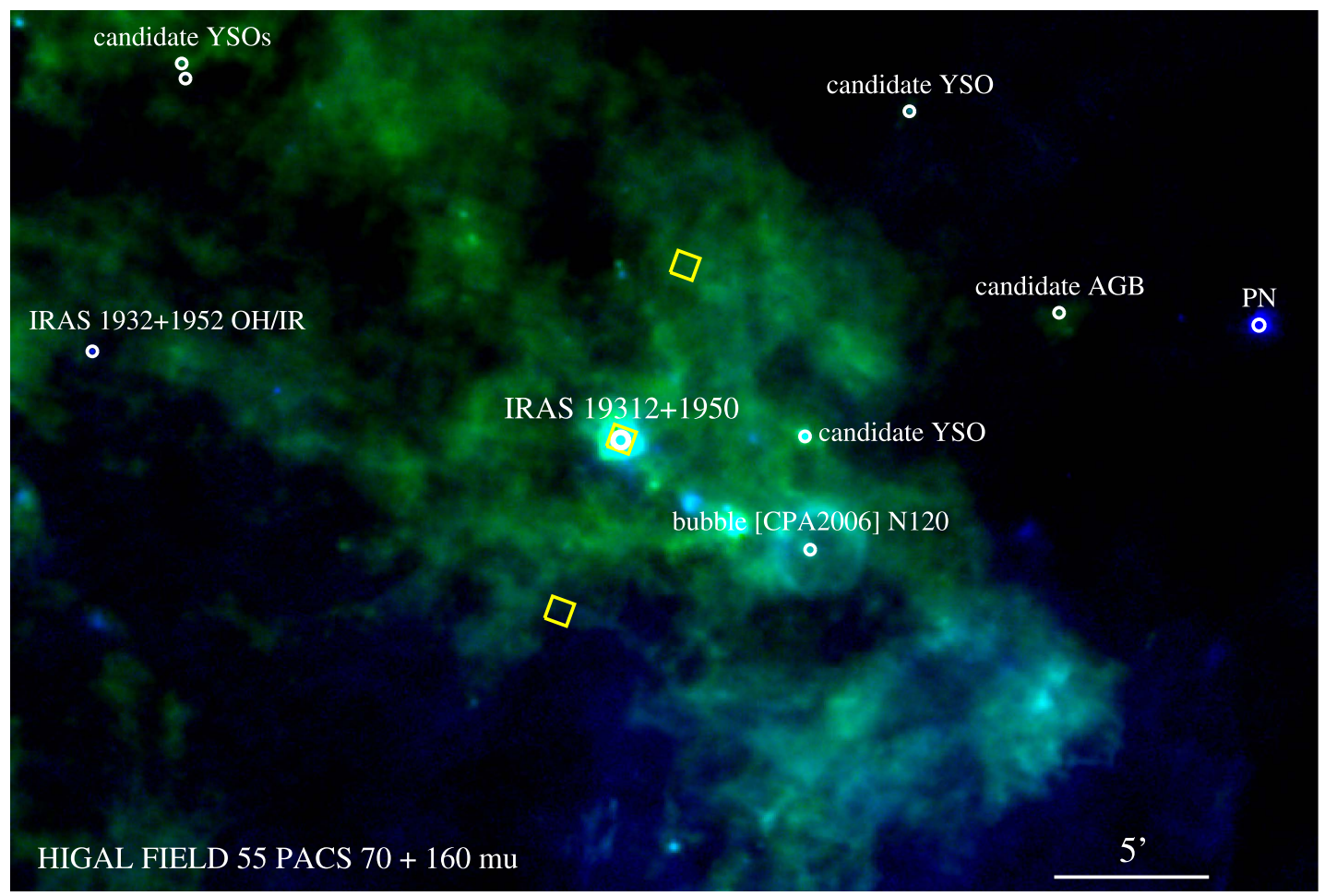

Figure 5. Herschel PACS two-color (green $=70 \mu \mathrm{m}$, blue $=160 \mu \mathrm{m}$ ) image of the region surrounding IRAS 19312+1950. Previously identified objects have been labeled. The on-source pointing position and PACS chop/nod reference positions are shown with yellow boxes.

have been derived. For ${ }^{13} \mathrm{CO}$ and $\mathrm{C}^{18} \mathrm{O}$, temperatures are found to be $81 \pm 3 \mathrm{~K}$, and $66 \pm 5 \mathrm{~K}$, respectively, confirming the presence of a gas component cooler than the $\sim 200 \mathrm{~K}$ component probed using PACS. Differing temperatures for these two $\mathrm{CO}$ isotopologues could be the result of optical depth effects in ${ }^{13} \mathrm{CO}$ (due to its larger abundance), which would act to reduce the $J=6-5$ line strength relative to $J=10-9$. A differing ${ }^{13} \mathrm{CO} / \mathrm{C}^{18} \mathrm{O}$ abundance ratio across varying excitation conditions in the source is also possible, for example, if the ${ }^{13} \mathrm{CO} / \mathrm{C}^{18} \mathrm{O}$ ratio is larger in a hotter region, which could result from isotopic variability in the wind of an evolved star.

Unfortunately, due to the relatively large HIFI beam size (see Table 2) and the complex source structure containing multiple kinematic components at differing temperatures, little can be inferred about the intrinsic properties of the source from beamaveraged, integrated spectral line fluxes. Regardless of the nature of IRAS $19312+1950$, the presence of excited CO and $\mathrm{H}_{2} \mathrm{O}$ indicates an intense source of energy. Emission from the higher-energy molecular transitions likely originates in a relatively compact, hot region close to the source, whereas lower-energy transitions trace cooler matter further away. Given the uncertain nature of the source, the detailed radiative transfer modeling required for the interpretation of the HIFI line fluxes is beyond the scope of the present article.

\subsubsection{HIFI Line Profiles}

The $\mathrm{CO}$ and $\mathrm{H}_{2} \mathrm{O}$ lines in Figure 6 (observed using HIFI WBS) show a wealth of spectral structure, particularly in the lower- $J$ transitions, which trace cooler, more optically thick gas. The CO $J=6-5$ and $J=5-4$ lines exhibit a broad component that rises to a narrow, double-peaked structure near the systemic velocity, indicative of self absorption in the line cores. The likelihood of $\mathrm{CO}$ self absorption is confirmed by the fact that the central dip coincides with the peak velocities of the less optically thick $\mathrm{C}^{18} \mathrm{O}$ and ${ }^{13} \mathrm{CO}$ lines (at $v_{L S R}=36.2$ $\mathrm{km} \mathrm{s}^{-1}$, shown with a dashed vertical line in Figure 6).

The low energy $\left(J^{\prime \prime}=1\right)$ transitions of $\mathrm{H}_{2} \mathrm{O}$ also exhibit a very broad component which, ignoring the presence of the narrow absorption features, has a relatively smooth and flattopped, Gaussian-like profile. Meanwhile, the less optically thick $\mathrm{H}_{2} \mathrm{O} 3_{12}-3_{03}$ line has an overall line shape very similar to CO $J=6-5$, for which the broad wings blend smoothly into the narrow line core. Such a "continuous" line profile may be indicative of a continuum of dynamical conditions surrounding IRAS $19312+1950$, in which a high-velocity outflow mixes gradually into a more quiescent (slowly moving or static) envelope.

The observed HIFI WBS spectra benefit from extremely clean, flat baselines, and emission is detectable far from the line cores: $\mathrm{CO} J=6-5$ flux is detected over the range -65 to $120 \mathrm{~km} \mathrm{~s}^{-1}$ (full width at zero intensity, FWZI $=185 \mathrm{~km} \mathrm{~s}^{-1}$ ) and $\mathrm{H}_{2} \mathrm{O} \quad 1_{10^{-}}-0_{01}$ spans -30 to $105 \mathrm{~km} \mathrm{~s}^{-1}$ $\left(F W Z I=135 \mathrm{~km} \mathrm{~s}^{-1}\right)$. Both ranges are approximately symmetrical about the $36 \mathrm{~km} \mathrm{~s}^{-1}$ systemic velocity, although the broad, Gaussian-like components of $\mathrm{H}_{2} \mathrm{O}$ and $\mathrm{CO}$ are centered at around $33 \mathrm{~km} \mathrm{~s}^{-1}$ (blueshifted from the $\mathrm{C}^{18} \mathrm{O}$ peak), indicating asymmetry in the outflow.

As pointed out by Deguchi et al. (2004), the broad line components imply the presence of a powerful wind emanating from the central star. However, the smooth, broad line profiles observed by HIFI lack the characteristic double-peaked/flattopped shape associated with (spherical) AGB-star outflows (see, for example, De Beck et al. 2012; Danilovich et al. 2014; Khouri et al. 2014). Instead their profiles are more similar to those observed toward low and high-mass protostars, where broad line wings are an observational characteristic of shocked 


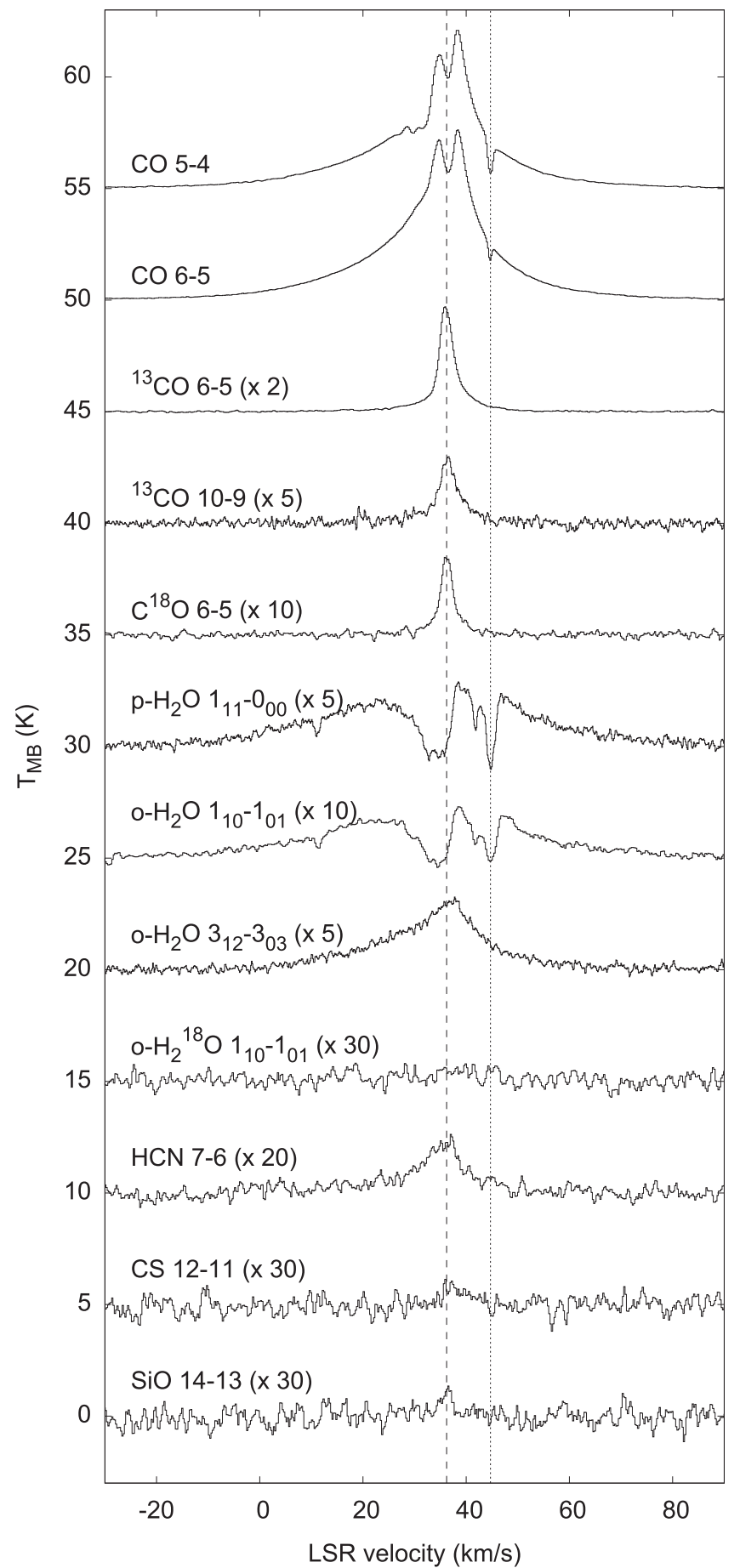

Figure 6. HIFI WBS molecular line observations of IRAS $19312+1950$. Spectra are baseline-subtracted and have multiplicative scaling (in parentheses), and additive offsets for display. Dashed vertical line shows the $\mathrm{C}^{18} \mathrm{O}$ $J=6-5$ peak velocity of $36.2 \mathrm{~km} \mathrm{~s}^{-1}$. Dotted line indicates a foreground absorption component.

gas accelerated by a fast bipolar outflow (Kristensen et al. 2010, 2012; San José-García et al. 2016). Indeed, our observed $\mathrm{H}_{2} \mathrm{O} 3_{12}-3_{03}$ line has FWZI $=64 \mathrm{~km} \mathrm{~s}^{-1}$, which is consistent with the mean value of $71 \pm 35$ observed by San José-García et al. (2016) in a sample of 19 high-mass YSOs using HIFI. Evolved stars, by contrast, typically have smaller $\mathrm{H}_{2} \mathrm{O}$ line FWZI in the range $10-45 \mathrm{~km} \mathrm{~s}^{-1}$ (e.g., Hunter et al. 2007).
An approximate temperature for the shocked/outflowing $\mathrm{CO}$ can be obtained from the ratio of the $J=6-5$ versus 5-4 line wing intensities. Excluding the optically thick line core region between $19-48 \mathrm{~km} \mathrm{~s}^{-1}$, a flux ratio of 1.30 was obtained, which corresponds to an excitation temperature of $\sim 330 \mathrm{~K}$ for the accelerated gas. This is roughly consistent with the $\sim 300 \mathrm{~K}$ rotational temperature of $\mathrm{CO}$ commonly observed by Herschel in shocked protostellar outflow cavity walls (Karska et al. 2014; Mottram et al. 2014; San José-García et al. 2016).

A CO absorption component occurs at $44.7 \mathrm{~km} \mathrm{~s}^{-1}$ (with FWHM $=0.4 \mathrm{~km} \mathrm{~s}^{-1}$ ), indicated with a dotted vertical line in Figure 6, and is possibly due to foreground interstellar gas. This foreground component shows up as a strong absorption feature in the ground-state (ortho and para) $\mathrm{H}_{2} \mathrm{O}$ transitions, but not in the higher-energy $3_{12}-3_{02}$ line, indicating a relatively low temperature $(\lesssim 100 \mathrm{~K})$. Similar to $\mathrm{CO}$, the ground-state $\left(J^{\prime \prime}=1\right) \mathrm{H}_{2} \mathrm{O}$ lines show strong absorption features at around the systemic velocity, but with peak self absorption offset to the blue, consistent with emission from a warm, inner region and the presence of cooler, outflowing (blueshifted) gas in our line of sight.

Despite a comparable peak line intensity and similar upperstate excitation energy, the $\mathrm{HCN} 7-6$ line is significantly broader than the $\mathrm{C}^{18} \mathrm{O} 6-5$ line $\left(\mathrm{FWHM}=13 \mathrm{~km} \mathrm{~s}^{-1}\right.$ versus $3.0 \mathrm{~km} \mathrm{~s}^{-1}$ ), indicating a larger abundance of $\mathrm{HCN}$ than $\mathrm{CO}$ in the accelerated gas. This indicates more efficient production of $\mathrm{HCN}$ than $\mathrm{CO}$ in the outflowing/shocked material.

The higher-resolution HIFI HRS spectra are nearly identical to the WBS spectra; the only significant difference is a slight increase in the depth of the narrow absorption component at $44.7 \mathrm{~km} \mathrm{~s}^{-1}$. The HRS spectra are not shown in the present article but are available for download from the Herschel Science Archive. ${ }^{10}$

\subsection{Spitzer Spectra}

The Spitzer IRS spectrum of IRAS 19312+1950 (covering the range $5-35 \mu \mathrm{m}$ ) is shown in the top panel of Figure 7 . The strongest features, at about $50 \%$ and $15 \%$, relative to the continuum, are seen near $9.7 \mu \mathrm{m}$ and $15 \mu \mathrm{m}$ and correspond to absorption by amorphous silicates and to the bending vibrational mode of $\mathrm{CO}_{2}$ ice, respectively. The silicate band may well be affected by emission at the longer wavelengths, as can be seen through comparison with the spectrum of the lowmass YSO RNO 91 between 10-12 $\mu \mathrm{m}$.

Other, weaker features visible in the Spitzer IRS spectrum are an emission band near $12.9 \mu \mathrm{m}$, an absorption near $5.8 \mu \mathrm{m}$, and structure in the $6.8-7.8 \mu \mathrm{m}$ wavelength range, all at around the $\approx 7 \%$ level relative to the continuum. The $5.8 \mu \mathrm{m}$ absorption feature is attributed to the bending mode of $\mathrm{H}_{2} \mathrm{O}$ ice. This band is commonly observed at $6.0 \mu \mathrm{m}$ toward embedded YSOs, and the shift to shorter wavelengths indicates that much of the absorption on the long-wavelength side of this ice feature is filled in with emission, for example, possibly due to the $\mathrm{C}-\mathrm{C}$ stretching mode by polycyclic aromatic hydrocarbons. The structure between $6.8-7.8 \mu \mathrm{m}$ is most likely real, but at the relatively low resolution of Spitzer IRS, it is unclear what the continuum level is, and thus whether the structure is caused by unresolved emission lines or absorption features. An (unidentified) emission feature appears to be present at $12.9 \mu \mathrm{m}$, although the longer-wavelength portion of this feature

$\overline{{ }^{10} \text { Herschel.esac.esa.int/Science_Archive.shtml }}$ 

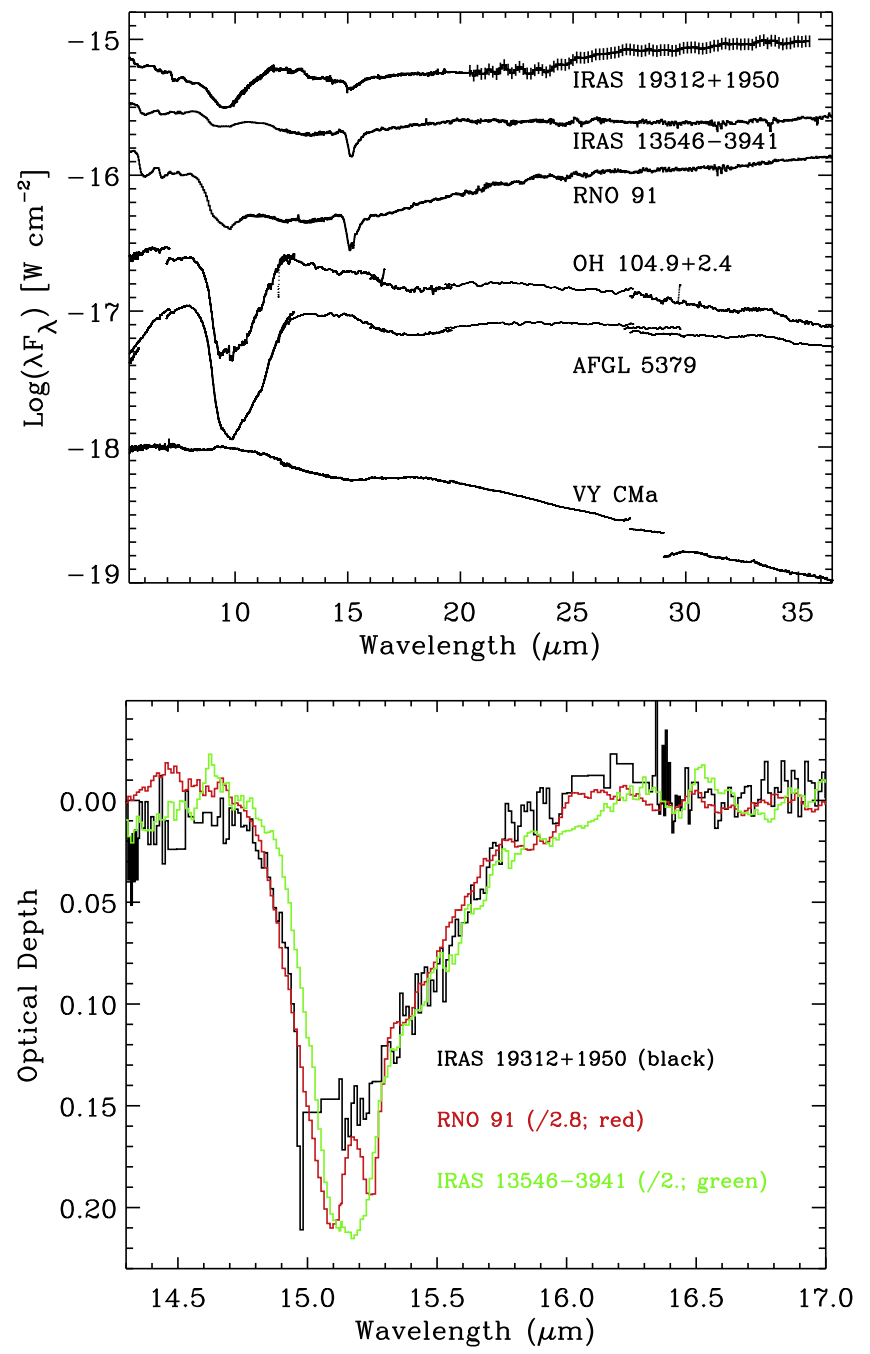

Figure 7. Top: Spitzer IRS spectrum of IRAS 19312+1950 (top trace), compared with spectra of other YSOs (IRAS 13546-3941 and RNO 91) and evolved stars (OH 104.9+2.4, AFGL 5379 and VY CMa) from Boogert et al. (2008), Sylvester et al. (1999), and Harwit et al. (2001). The $1 \sigma$ error bars are shown for IRAS $19312+1950$, which only become significant at wavelengths $>20 \mu \mathrm{m}$ due to detector saturation. Bottom: Zoomed region surrounding the $15 \mu \mathrm{m} \mathrm{CO}_{2}$ ice band of IRAS $19312+1950$, compared with scaled spectra of other YSOs (overlaid in red and green).

has been masked out due to saturation of the detector array. In general, to further study these weaker features, higher spectral resolution $(R \gtrsim 1000)$ observations, unaffected by array saturation, are needed. Features longward of $20 \mu \mathrm{m}$ are within the noise envelope so probably not real.

Crystalline $\mathrm{H}_{2} \mathrm{O}$ ice absorption bands have been observed in high mass-loss, dense, evolved stellar envelopes exhibiting $\mathrm{OH}$ maser emission, and also in the expanding, cool envelopes of slightly more evolved post-AGB stars (Boogert et al. 2015). On the other hand, $\mathrm{CO}_{2}$ ice is not seen in evolved stellar envelopes. For example, toward the OH/IR star AFGL 5379 (also shown in the top panel of Figure 7), the $3.0 \mu \mathrm{m} \mathrm{H}_{2} \mathrm{O}$ ice band has a peak optical depth of $\sim 0.7$ (Sylvester et al. 1999), which translates to a $\mathrm{H}_{2} \mathrm{O}$ column density of $1.2 \times 10^{18} \mathrm{~cm}^{-2}$ (using an integrated band strength of $2 \times 10^{16} \mathrm{~cm}^{-2}$ and a FWHM of $330 \mathrm{~cm}^{-1}$ ). Toward massive YSOs, the $\mathrm{CO}_{2} / \mathrm{H}_{2} \mathrm{O}$ column density ratio is typically about $19 \%$, and the lowest observed value is $11 \%$ (Gerakines et al. 1999). Hence, if evolved stars had ISM-like ice abundances, then a $\mathrm{CO}_{2}$ column density of

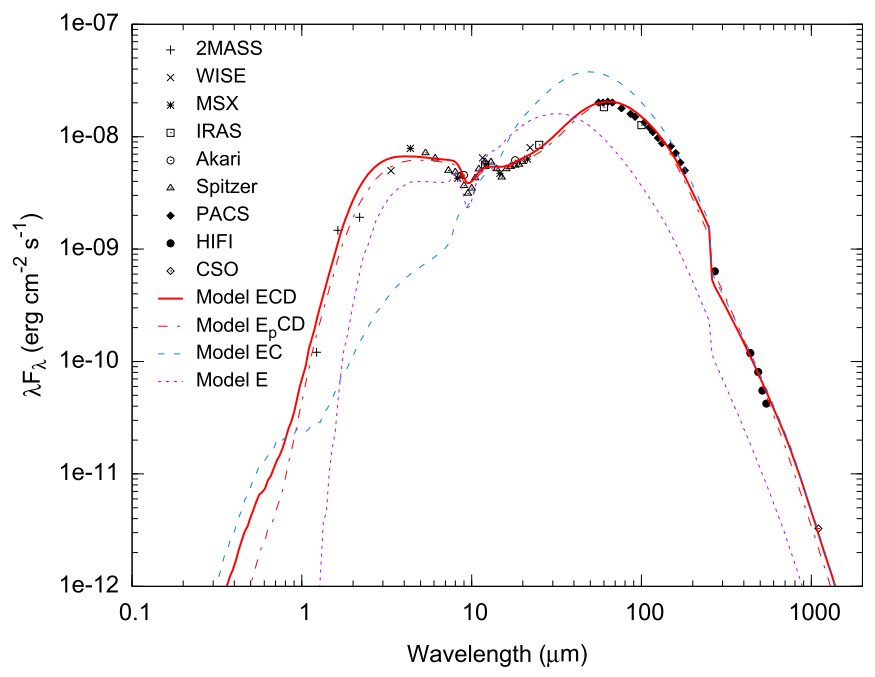

Figure 8. IRAS $19312+1950$ spectral energy distribution compiled from various telescope observations as shown in the key. Four least-squares YSO SED model fits are overlaid: (1) rotationally flattened collapsing envelope (E), (2) rotationally flattened collapsing envelope with bipolar cavity (EC), (3) rotationally flattened collapsing envelope with bipolar cavity and disk (ECD), and (4) power-law envelope with bipolar cavity and disk $\left(\mathrm{E}_{p} \mathrm{CD}\right)$. Parameters for the best-fitting SED model (using the analytical YSO model of Whitney et al. 2003) are given in Table 6. Apparent discontinuity in the model SEDs near $250 \mu \mathrm{m}$ is due to the difference in aperture size for the PACS and HIFI observations.

$\gtrsim 1.2 \times 10^{17} \mathrm{~cm}^{-2}$ would be expected for AFGL 5379. Using observed band widths (Gerakines et al. 1999) and laboratorymeasured integrated band strengths (Gerakines et al. 1995), this would result in peak optical depths of 0.45 for the $4.2 \mu \mathrm{m} \mathrm{CO}_{2}$ stretch mode, and 0.05 for the $15 \mu \mathrm{m}$ bending mode. While this is at the noise level for the observed $15 \mu \mathrm{m}$ spectrum (Figure 7), it is a factor of 5 above any structure observed around $4.2 \mu \mathrm{m}$ (Sylvester et al. 1999), confirming the $\mathrm{CO}_{2}$-poor environment of $\mathrm{OH} / \mathrm{IR}$ shells. A similar situation is also true for the evolved O-rich star $\mathrm{OH} \mathrm{104.9+2.4} \mathrm{(also} \mathrm{shown} \mathrm{in} \mathrm{Figure} \mathrm{7).} \mathrm{We}$ therefore conclude that the $15 \mu \mathrm{m} \mathrm{CO}$ ice band observed toward IRAS 19312+1950 must originate from the envelope/ disk of a YSO or a foreground cloud.

For comparison, we also show an ISO spectrum of the high mass-loss red supergiant star VY CMa in the top panel of Figure 7 (see also Harwit et al. 2001). Silicate emission bands occur around 10 and $18 \mu \mathrm{m}$, and the SED falls steadily toward the far-IR, in contrast to the rising SED of IRAS 19312+1950. A close-up of the $15 \mu \mathrm{m}$ region oberved by Spitzer for IRAS $19312+1950$ and the YSOs RNO 91 and IRAS 135463941 is shown in the lower panel of Figure 7. The close correspondence between these $\mathrm{CO}$ ice band profiles confirms a similarity of the conditions in the envelope of IRAS 19312 +1950 to those around other YSOs.

\section{DUST EMISSION AND SED MODELING}

\subsection{Observational SED Data}

Figure 8 shows a compilation of fluxes measured toward IRAS $19312+1950$ using various (predominantly space-based) telescopes; data from each instrument are plotted using a different symbol. Continuum fluxes were measured from the HIFI spectra, taken from regions adjacent to the spectral lines and free of any apparent line emission in either sideband (see Table 5). The measured HIFI fluxes are average values from 
Table 5

HIFI Continuum Fluxes

\begin{tabular}{lc}
\hline \hline$\lambda(\mu \mathrm{m})$ & Flux $(\mathrm{Jy})$ \\
\hline $537+549$ & 7.7 \\
$508+521$ & 9.5 \\
$484+491$ & 13.1 \\
$434+440$ & 17.4 \\
$269+272$ & 57.1 \\
\hline
\end{tabular}

Note. Fluxes are average values for two wavelengths due to contributions from the two HIFI sidebands and have been converted from Kelvin to Jansky assuming a point source.

two frequencies separated by $\sim 10 \mathrm{GHz}$ due to the contributions from the two receiver sidebands.

The PACS fluxes were extracted from the reduced scanmaps using a 54"-diameter circular aperture surrounding the central source. The extracted spectra were then uniformly sampled in log-wavelength space, with wavelengths chosen to exclude gaseous emission features and regions of less certain PACS calibration toward the edges of the spectral bands. Despite using the most up-to-date PACS calibration data, slight discontinuities are evident between the different PACS SED spectral bands, although these are within the nominal absolute flux calibration accuracy of $10 \%$. Reliability of the PACS measurements is confirmed by their close correspondence (within 10\%) of the IRAS measurements at 60 and $100 \mu \mathrm{m}$. The Spitzer spectra were sampled at a wavelength interval of approximately $0.5 \mu \mathrm{m}$ below 11 and $1 \mu \mathrm{m}$ above, and fluxes are consistent with previous observations in the mid-IR. Infrared survey fluxes were taken from the most up-to-date data products from each observatory. The WISE $4.6 \mu \mathrm{m}$ flux point has been omitted due to detector saturation. The Herschel PACS and HIFI measurements from the present study lie toward the far right of the plot, sampling the longest wavelengths and filling in the region around the SED peak where data was previously lacking. The $1100 \mu \mathrm{m}$ data point comes from the Caltech Submillimeter Observatory Bolocam survey (Rosolowsky et al. 2010).

The resulting SED (plotted logarithmically in units of uniform energy density, $\lambda F_{\lambda}$ ) shows a broad maximum near $64 \mu \mathrm{m}$, consistent with thermal emission from cold dust. The $9.7 \mu \mathrm{m}$ silicate absorption peak is evident, and a secondary peak around $3-5 \mu \mathrm{m}$ is probably due to hot dust in the vicinity of the central source. Fluxes fall rapidly toward the near-IR, and IRAS $19312+1950$ is extremely faint at optical wavelengths. The double-peaked SED is characteristic of deeply embedded YSOs (e.g., Adams et al. 1987; Robitaille et al. 2006; Grave \& Kumar 2009), whereas evolved star SEDs tend to fall off much more rapidly toward the far-IR (see, for example Sylvester et al. 1999; Harwit et al. 2001; Povich et al. 2009; Danilovich et al. 2014).

\subsection{SED Modeling}

Preliminary fitting of the SED was performed using the grid of 200,000 theoretical YSO models published by Robitaille et al. (2006). These SED models consist of four main components: (1) a rotationally flattened, collapsing circumstellar envelope, (2) a pair of low-density bipolar outflow cavities, (3) a flared Keplerian disk, and (4) a central (blackbody) radiation source. The model parameters span a broad range of stellar temperatures, luminosities, ages, disk,

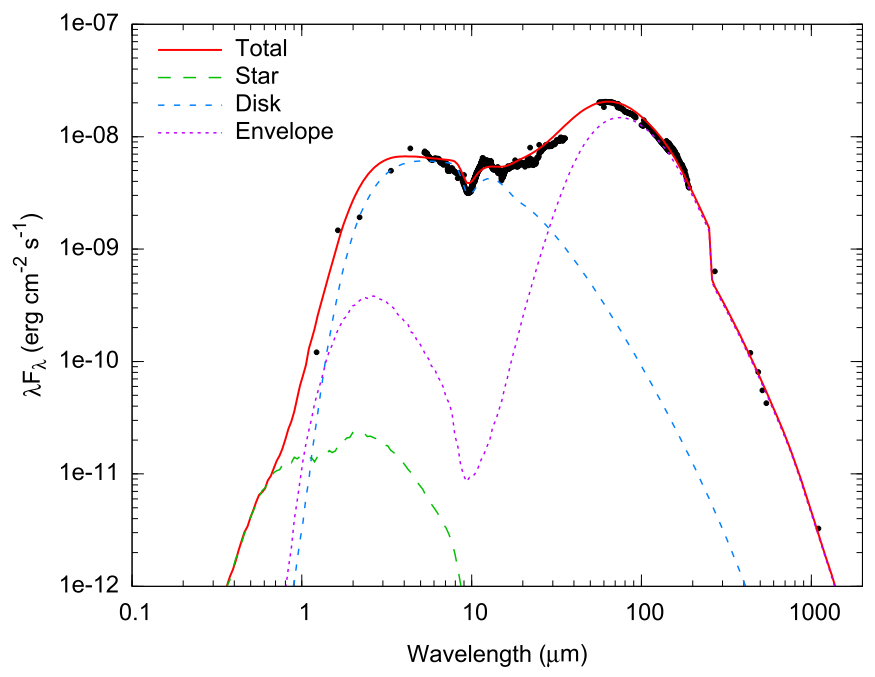

Figure 9. IRAS $19312+1950$ spectral energy distribution with best-fitting (ECD) model of Figure 8 overlaid (red solid curve). Contributions to the total SED from the (reddened) central star, the disk and the envelope are shown with dashed curves. Apparent discontinuity in the model SED near $250 \mu \mathrm{m}$ is due to the difference in aperture size for the PACS and HIFI observations.

outflow, and envelope properties. Additional details of the physical model and its parametrization are given by Whitney et al. (2003).

The best-fitting YSO model (\#3017279) was re-computed using the HYPERION 3D Monte Carlo dust radiative transfer code (Robitaille 2011). The Kim, Martin, \& Hendry dust opacity model was used, with a dust sublimation temperature of $1600 \mathrm{~K}$. The number of photons was set to $10^{6}$ for the specific energy calculation and $10^{7}$ for peeling-off of the SED fluxes as a function of wavelength. The modified random walk approximation (with $\gamma=1$ ) was employed for regions of very high optical depth in the disk mid-plane. The final SED model was calculated using an azimuthally symmetric polar grid with 200 (altitudinal) angular cells and 500 radial cells, with logarithmic distance increments.

Due to the decreasing dust temperature with distance from the source, the apparent circumstellar envelope size increases with wavelength and starts to become significantly larger than the adopted 54" PACS aperture for wavelengths $\gtrsim 70 \mu \mathrm{m}$. For comparison with the PACS data, model SED fluxes were therefore calculated in HYPERION using a 54" aperture; for other observations longward of $200 \mu \mathrm{m}$, an aperture size corresponding to the respective telescope's half-power beamwidth (HPBW) was used. For observations at shorter wavelengths, the entire model flux was used (i.e., employing an aperture of infinite size).

Nonlinear least-squares optimization of the YSO model parameters with respect to the observed SED was performed using the MPFIT routine (Markwardt 2012). The distance, inclination angle and foreground extinction were also optimized. The resulting best-fit SED model is shown in Figure 8 (solid red curve), and the corresponding parameter values are given in Table 6. The model density and temperature distributions are shown in Figure 10.

\subsection{SED Model Variations}

The requirement of a three-component dust model consisting of an envelope, a bipolar cavity, and a disk (abbreviated ECD) was examined by running two additional models: (1) including 
Table 6

Best-fitting SED Model Parameters

\begin{tabular}{lc}
\hline \hline Parameter & Value \\
\hline Stellar luminosity & $17,000 \mathrm{~L}_{\odot}$ \\
Stellar mass & $9.6 \mathrm{M}_{\odot}$ \\
Disk mass & $0.21 \mathrm{M}_{\odot}$ \\
Disk radius & $116 \mathrm{au}$ \\
Disk scale-height at $100 \mathrm{au}$ & $17 \mathrm{au}$ \\
Disk density exponent & -0.8 \\
Disk flaring power & 1.1 \\
Envelope infall rate & $\times 10^{-3} \mathrm{M}_{\odot} \mathrm{yr}^{-1}$ \\
Centrifugal radius & $130 \mathrm{au}$ \\
Envelope radius & $1.1 \times 10^{5} \mathrm{au}$ \\
Cavity opening angle & $81^{\circ}$ \\
Inclination angle & $48^{\circ}$ \\
Total mass (gas + dust) & $730 M_{\odot}$ \\
Distance & $3300 \mathrm{pc}$ \\
Foreground extinction & $3.7 \mathrm{mag}$ \\
Total visual extinction & $72 \mathrm{mag}$ \\
\hline
\end{tabular}

only an envelope and cavity (EC) and (2) including only the envelope (E). The parameters in these models were subject to the same least-squares optimization as the full ECD model, to arrive at the SEDs plotted using dashed curves in Figure 8. The ECD model represents a good fit to the data, whereas the $\mathrm{E}$ and EC models are unable to match the observations, thus demonstrating the requirement of (at least) three dust components. The simplest model (E) is unable to simultaneously reproduce both the mid and far-IR SED peaks because there is no easy route for radiation to escape and the outer envelope becomes too hot, which shifts the SED peak to the blue. Adding a bipolar outflow cavity with no disk (EC) allows too much radiation to escape from the inner envelope, which is then too cool to reproduce the secondary peak at around $5 \mu \mathrm{m}$. The presence of a dense, inner disk-type structure is required to resolve this discrepancy. To highlight the relative contributions of the three main sources of flux, the separate contributions to the SED from the central star, the disk, and the envelope (including both directly emitted and scattered radiation) are shown in Figure 9.

The final SED model fits the data remarkably well apart from in the mid-IR (between 2 and $4 \mu \mathrm{m}$ ). Discrepancies in this region could be due to deficiencies in the adopted (azimuthally symmetric) flared disk density distribution. Alternatively, clumpy structure in the outflow cavity, or the presence of companion star(s) could produce additional sources of opacity or emission in this range.

The hot (up to $\sim 1600 \mathrm{~K}$ ), dense (up to $\sim 10^{12} \mathrm{~cm}^{-3}$ ), dusty disk residing between about 20 and 150 au from the central star provides the main source of flux in our model between 2-20 $\mu \mathrm{m}$. However, given the slight mismatch between model and observations at shorter wavelengths, the shape of the disk is certainly not well constrained. Indeed, although a mass of hot, dense dust seems to be required, it may be possible to reproduce this part of the SED without employing a classical disk-like structure. Accordingly, the analytical YSO model (with parameters given in Table 6) probably does not represent a unique solution for the structure of the dust distribution. In reality, azimuthal symmetry is unlikely and the structure of the envelope, disk and outflow may be clumpy and turbulent, with various infalling streams and outward-moving jets of material (as suggested by the structures in Figure 1). In addition, the ad hoc nature of the Whitney et al. (2003) YSO model creates an artificial (and non-physical) discontinuity at the disk boundaries. Exploring the complete parameter space of physically plausible dust distributions that can reproduce the observed SED may be worthwhile in the future once the region around $1-5 \mu \mathrm{m}$ is better characterized, but such an analysis is beyond the scope of the present study. The most important parameters here are the intrinsic luminosity of the central source, as well as the mass and size of the envelope, which are well constrained by our models. The implied presence of a warm inner disk could be tested through future high-resolution sub-millimeter molecular line observations.

To further assess the uniqueness of the best-fitting SED model parameters, the rotationally flattened infalling envelope structure of Ulrich (1976), which has a radial density dependence of $\sim r^{-1.5}$, was replaced by a simple spherically symmetric envelope with an $r^{-\alpha}$ density profile (with $\alpha$ as a free parameter). This model was optimized as in Section 4.2, and the best-fitting parameters resulted in an envelope mass of $470 M_{\odot}$, density exponent $\alpha=2.2$, and source luminosity of $20,000 L_{\odot}$. The SED corresponding to this "power-law envelope + disk + cavity" model is shown with a dot-dashed style in Figure 8 (and labeled $\mathrm{E}_{p} \mathrm{CD}$ ). The greater luminosity of this model is primarily due to a slightly larger best-fitting distance of $3.6 \mathrm{kpc}$. Other parameters such as the disk mass, size, and shape and the cavity opening angle and orientation are not significantly different from the best-fitting ECD model parameters in Table 6. Given the known distance of IRAS $19312+1950$ from $\mathrm{H}_{2} \mathrm{O}$ maser parallax observations $\left(3.8_{-0.58}^{+0.83} \mathrm{kpc}\right.$; Imai et al. 2011), the total luminosity is well constrained to the range $L=16,000-25,000 L_{\odot}$.

A blackbody temperature for the central source in the range $T \sim 10,000-25,000 \mathrm{~K}$ provides the best fit to the SED. Lower temperatures result in a worse fit in the $20-30 \mu \mathrm{m}$ region, but cannot be ruled out given the lack of observational constraints on the structure of the disk and inner envelope. Indeed, if IRAS $19312+1950$ is a YSO, then a relatively low source temperature (and young evolutionary stage) is implied by the non-detection of free-free radio emission. Using the formula of Carpenter et al. (1990), the $0.66 \mathrm{mJy} 3 \sigma$ upper limit of the CORNISH $5 \mathrm{GHz}$ survey (Purcell et al. 2013) corresponds to a total ionizing photon flux of $10^{45} \mathrm{~s}^{-1}$, which suggests a relatively small $\mathrm{H}$ II region with a stellar effective temperature $\lesssim 10,000 \mathrm{~K}$.

Our best-fitting SED model differs significantly from that of the AGB-envelope model of Murakawa et al. (2007), who derived $L=7000 L_{\odot}$ for an adopted source distance of $2.5 \mathrm{kpc}$. The discrepancy between our models is primarily due to improvements in the characterization of the far-infrared fluxes by Spitzer and Herschel, but also to the improved distance estimate from Imai et al. (2011). Although Murakawa et al. (2007) took the IRAS far-IR fluxes at 60 and $100 \mu \mathrm{m}$ into account in their modeling, they assumed a large background contamination (and hence, a large uncertainty) for those measurements. Our Herschel images demonstrate that the assumption of a strong far-IR background was erroneous, as shown in Figures 3 and 5 where IRAS 19312+1950 appears as a spatially isolated object. Taking the average background flux from the Hi-Gal $70 \mu \mathrm{m}$ image using an annulus between 150"$200^{\prime \prime}$ from the center of the source gives a possible background flux contribution of only $3.4 \%$ for our measured far-IR continuum fluxes. Furthermore, as shown in Figure 5, the PACS reference positions (yellow squares) covered regions 

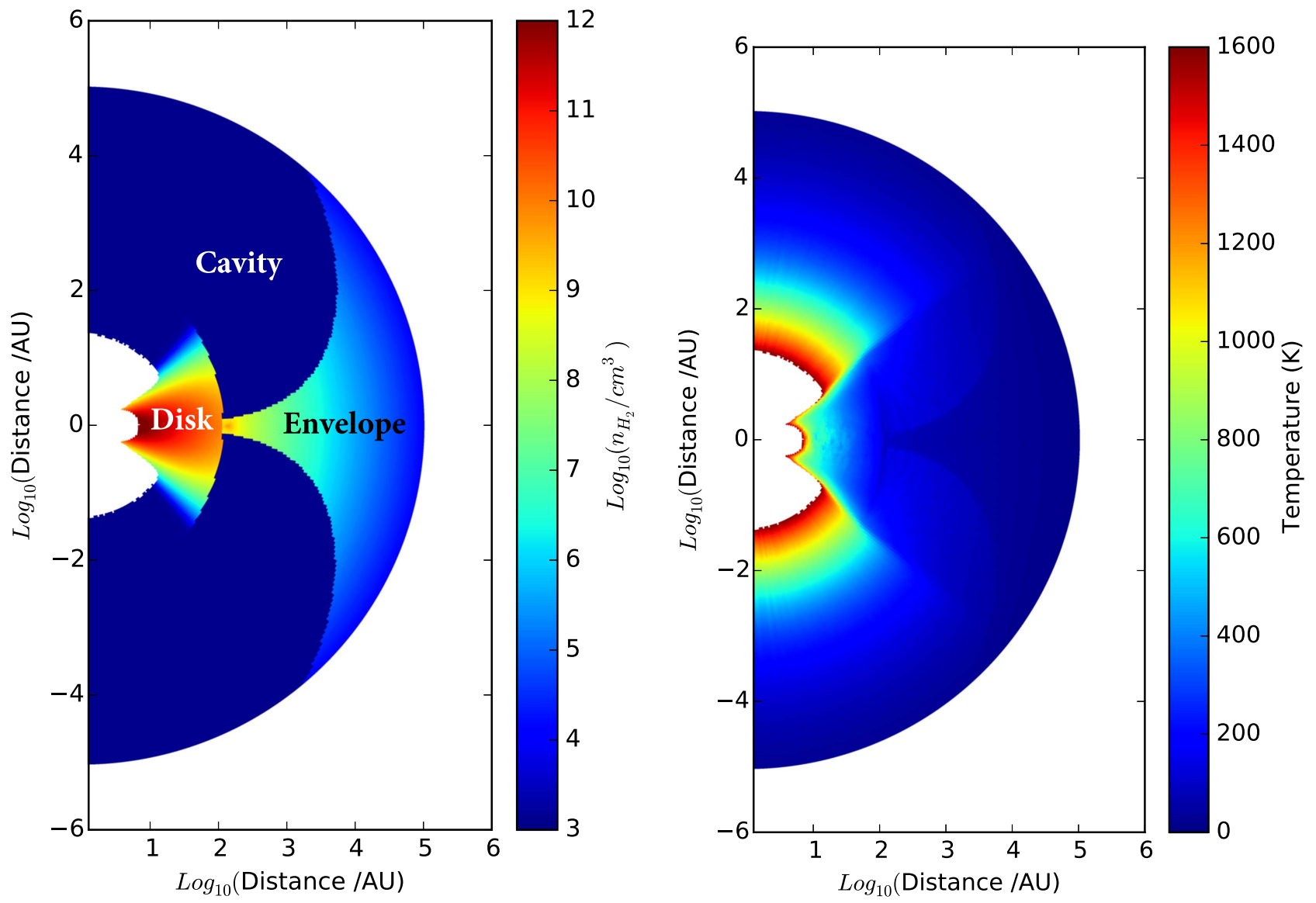

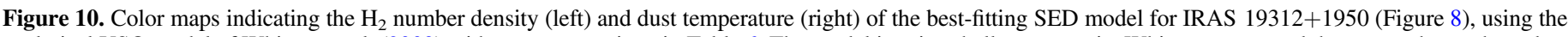

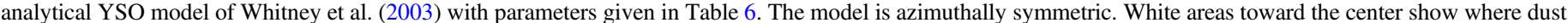

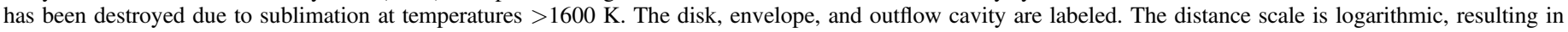
apparent distortion of the cavity shape.

with a similar level of background emission to that near the central source, thus providing an approximate (serendipitous) background correction for the source signal and negating the need for additional corrections.

\section{DISCUSSION}

The circumstellar envelope mass of $\sim 500-700 M_{\odot}$ derived by modeling the far-IR SED compares reasonably well with the $225-478 M_{\odot}$ obtained from CO observations by Nakashima et al. (2016), and is much too large to have originated from the outflow of an evolved star. Nakashima et al. (2016) derived their envelope mass assuming a standard interstellar gas-phase $\mathrm{CO} / \mathrm{H}_{2}$ ratio of $8 \times 10^{-5}$, and so their total mass estimate would be correspondingly larger if a fraction of the $\mathrm{CO}$ is frozen out onto dust (which is usually the case in massive starforming cores; Rygl et al. 2013). Deguchi et al. (2004)'s original envelope mass estimate of only $25 M_{\odot}$ (from $\mathrm{CO}$ $J=1-0$ line modeling, assuming a $r^{-3}$ density distribution between $r=5-15^{\prime \prime}$ ) appears to have severely underestimated the amount of gas surrounding IRAS 19312+1950. A clump mass of $1200 \pm 200 M_{\odot}$ was obtained by Dunham et al. (2011) using $1.1 \mathrm{~mm}$ continuum and $\mathrm{NH}_{3}$ radio spectroscopic observations of IRAS 19312+1950 (assuming a spherical source with constant temperature and density), which provides further evidence for a large total mass of circumstellar material. In our best-fitting SED model, circumstellar material contributes $\sim 70$ mag toward the total line-of-sight visual extinction of the central star. Such high extinction implies that the majority of the observed $\mathrm{CO}_{2}$ and $\mathrm{H}_{2} \mathrm{O}$ ice absorption occurs in the circumstellar envelope rather than in foreground interstellar material.

The stellar luminosity and mass derived as independent parameters from our SED fitting are consistent with the premain-sequence (PMS) evolutionary track of an early B-type supergiant star (e.g., Hernández et al. 2004; Elia et al. 2010). Based on massive YSO accretion models, the large luminosity and intermediate envelope mass would place IRAS 19312 +1950 close to (within a few times $10^{4}$ years of) the end of its main accretion phase (see for example Molinari et al. 2008). Such high luminosities are rarely observed in massive evolved stars due to the very rapid evolution during the final stages of their lifetimes (see Figure 2 of van Winckel 2003); a $7 M_{\odot}$ post-AGB star is predicted to expel its envelope in only a few hundred years, although thermal pulses can prolong this stage.

The average $\mathrm{CO}$ temperature observed with PACS of $T=197.7 \pm 2.1 \mathrm{~K}$, and outflow temperature from HIFI of $\sim 330 \mathrm{~K}$, are consistent with the $\sim 300 \mathrm{~K}$ temperatures observed in the the outflow cavity walls of low- and high-mass protostars derived in other Herschel studies (e.g., Karska et al. 2014; Mottram et al. 2014; San José-García et al. 2016). The broad, Gaussian-type line wings observed in our $\mathrm{H}_{2} \mathrm{O}$ and $\mathrm{CO}$ HIFI spectra are also highly characteristic of shocked outflowing protostellar gas. San José-García et al. (2016) found that the majority of $\mathrm{H}_{2} \mathrm{O} 3_{12}-3_{03}$ line emission from massive YSOs originates in shock-accelerated gas along the cavity walls. In 
addition to the close similarity in the $\mathrm{H}_{2} \mathrm{O} 3_{12}-3_{03}$ line profile between IRAS $19312+1950$ and other protostars, the integrated line luminosity of $L_{\mathrm{H}_{2} \mathrm{O}}=2.0 \mathrm{~K} \mathrm{~km} \mathrm{~s}^{-1} \mathrm{pc}^{2}$ (combined with a source luminosity of $\sim 20,000 L_{\odot}$ ) puts IRAS 19312 +1950 precisely in line with the $L_{\mathrm{H}_{2} \mathrm{O}}$ versus $L_{\text {bol }}$ trend observed in 32 protostars by San José-García et al. (2016). Similarly, the CO $J=14-13$ and O I $146 \mu \mathrm{m}$ line luminosities (0.60 and $0.23 L_{\odot}$, respectively) are consistent with the close correlations between line luminosity and stellar luminosity observed by Karska et al. (2014) in a large sample of protostars spanning the range from low to high mass. Thus, the same conclusion regarding the nature of IRAS $19312+1950$ is reached through three separate lines of analysis: (1) the lowresolution far-IR spectrum, (2) the high-resolution HIFI line profiles, and (3) the infrared SED, which are all consistent with a luminous protostar embedded in a massive, dusty envelope, and powering a fast bipolar outflow. The power-law envelope density structure that provides a good fit to the SED of IRAS $19312+1950$ is a common feature of actively accreting protostars (Keto et al. 2015; Tobin et al. 2015). Based on our interpretation of the data, the idea that a massive, evolved star found its way to the center of a collapsing molecular cloud during its brief, final, high-luminosity phase is highly unlikely.

Two temperature regimes are seen in our PACS CO observations. The warmest component (at $280 \mathrm{~K}$ ) has a mass of around $0.22 M_{\odot}$, which is similar to the mass of the disk required to match the mid-IR continuum emission in our bestfitting SED model. The cooler $(160 \mathrm{~K})$ component is associated with $\approx 1.6 M_{\odot}$ of material, which might be representative of shocked or radiatively heated gas in the outflow cavity walls further from the star. This hypothesis could be examined by mapping the thermal and kinematic structure through spectrally and spatially resolved observations of high-excitation CO lines. Such observations would be invaluable as a probe of the energetic feedback processes between star and envelope, and would facilitate understanding the nature of outflow shocks in massive YSOs.

Our new understanding of IRAS $19312+1950$ is also consistent with the previous molecular line observations of this source. The $\sim 2 \times 10^{5}$ au $\left(\sim 50^{\prime \prime}\right.$ diameter $)$ dense, dusty circumstellar envelope lies within the boundaries of a $\sim 100^{\prime \prime}$ wide $\mathrm{CO}$ cloud detected at the same position by Nakashima et al. (2016). Broad ( $\sim 50 \mathrm{~km} \mathrm{~s}^{-1}$ wide) components in the $\mathrm{CO}$ $J=1-0$ and 2-1 lines observed by Nakashima \& Deguchi (2005) are consistent with the outflow wings we observed using HIFI, although these were previously interpreted as arising in a spherically expanding (AGB star) outflow. It was noted by Nakashima \& Deguchi (2005), however, that their line profiles were rather inconsistent with the flat-topped shape expected for a spherical outflow. In the collapsing protostellar envelope paradigm, the Nakashima \& Deguchi (2005) CO observations may be best explained by a wide-angle bipolar outflow. If the outflowing gas decelerated as it expanded into the ambient envelope, then this would be consistent with the observation of a spatially compact, high-velocity $\mathrm{CO}$ component that gradually transitions to slower, more extended components with distance from the central star. This explanation may be consistent with the smooth transition from high-velocity wind to quiescent (at rest) envelope, manifested in our HIFI ${ }^{12} \mathrm{CO}$ and ${ }^{13} \mathrm{CO} J=6-5$ line profiles. The narrow, $1-2 \mathrm{~km} \mathrm{~s}^{-1} \mathrm{CO}$ outflow component identified by Nakashima \& Deguchi (2005) could plausibly be probing a slower-moving part of the accelerated gas further out in the envelope. This is consistent with the 1-0 transition probing mostly cooler gas, which would be further from the shock front of the putative protostellar outflow. Alternatively, the low-velocity (narrow) red- and blueshifted CO components could originate in the receding and approaching hemispheres of a rotating, collapsing envelope or massive disk. Other high-velocity (broad) molecular line components observed by Deguchi et al. (2004) are readily explainable as arising from shocked gas from a protostellar envelope, swept up by an impinging fast bipolar outflow.

Two red and blueshifted $\mathrm{H}_{2} \mathrm{O}$ (and $\mathrm{SiO}$ ) maser components were observed toward IRAS $19312+1950$ by Nakashima et al. (2011). The $\mathrm{H}_{2} \mathrm{O}$ maser components are separated by about 11 mas along an axis $107^{\circ}$ counterclockwise from north, and interpreted as most likely arising in a bipolar outflow. Water maser activity is commonly associated with massive star formation (Churchwell et al. 1990) as well as outflows from evolved stars (Yoon et al. 2014). As shown by Torrelles et al. (1998), $\mathrm{H}_{2} \mathrm{O}$ masers can be associated with both protostellar outflows and disks. If the $\mathrm{H}_{2} \mathrm{O}$ (and $\mathrm{SiO}$ ) maser spots of IRAS $19312+1950$ are interpreted as arising in a Keplerian disk with its axis in the plane of the sky, then the velocity displacement of $35 \mathrm{~km} \mathrm{~s}^{-1}$ corresponds to a mass of $4.4-6.7 M_{\odot}$ for the central star (assuming a distance of $2.5-3.9 \mathrm{kpc}$; Nakashima et al. 2011). Accounting for a $48^{\circ}$ inclination to our line of sight (as derived from our SED modeling) yields a stellar mass range $6-9 M_{\odot}$ which is consistent with a massive protostar. Alternatively, due to the strongly linear distribution of $\mathrm{H}_{2} \mathrm{O}$ maser spots with a clear separation between the red and blue components, their association with a well-collimated bipolar outflow may be more likely. In this scenario, the apparent $\mathrm{N}-\mathrm{S}$ extension of our PACS spectral line and continuum images (Figure 3 ) could be representative of the size of the wide-angle cavity in a direction perpendicular to the outflow and partially oriented toward our line of sight. Additional high-resolution molecular mapping, as well as continued monitoring of the positions of the $\mathrm{H}_{2} \mathrm{O}$ maser spots, is needed in order to establish the direction of the bipolar outflow and disk axes to help resolve this dichotomy.

Previous radio studies have shown $18 \mathrm{~cm} \mathrm{OH}$ maser line properties to be useful diagnostics of YSOs and evolved stars (e.g., Herman \& Habing 1985; te Lintel Hekkert \& Chapman 1996; Caswell 1998; Edris et al. 2007). Accordingly, the strength of the $1612 \mathrm{MHz} \mathrm{OH}$ maser line relative to the 1665 and $1667 \mathrm{MHz}$ lines observed by Nakashima et al. (2011) was taken as good evidence that IRAS $19312+1950$ is an evolved star and not a YSO. Although the $1612 \mathrm{MHz} O H$ maser tends to appear most strongly in the expanding shells of high massloss oxygen-rich AGB stars (David et al. 1993), it can also be found in massive YSOs (e.g., Cohen et al. 2006; Ramachandran et al. 2006). The precise physical conditions required for pumping this transition are not yet fully understood (Fish et al. 2006), and so a closer examination of the cause of its unusually strong occurrence in IRAS $19312+1950$ could help shed light on this maser's origin.

\section{CLASSIFICATION OF IRAS 19312+1950 AND COMPARISON WITH OTHER SOURCES}

Our new infrared observations of IRAS $19312+1950$ have revealed a far-IR spectrum of gas, dust, and ice highly consistent with a massive YSO in an early evolutionary stage. 
The hypothesis that the central radiation source could be an evolved star inside its natal molecular cloud (as suggested Nakashima et al. 2011, 2016) is deemed very unlikely. No such objects have been previously identified, which is consistent with the expectation that the protostellar envelope and natal cloud(s) would have dissipated and returned to the diffuse phase during the latter stages of star formation (Lada et al. 1987; McKee \& Ostriker 2007).

The possibility of a chance encounter between a massive, evolved star and a dense molecular cloud cannot be ruled out entirely. However, the red supergiant (RSG) scenario favored by Nakashima et al. (2016) seems unlikely due to the $\gtrsim 90$ $\mathrm{km} \mathrm{s}^{-1}$ outflow velocity implied by our HIFI CO observations. Terminal RSG wind speeds are typically $\sim 10-30 \mathrm{~km} \mathrm{~s}^{-1}$, and based on the relation of Mauron \& Josselin (2011), the relatively low luminosity of IRAS $19312+1950$ (compared to other RSGs) would imply a wind speed of only about $11 \mathrm{~km} \mathrm{~s}^{-1}$. More highly evolved post-AGB stars (such as $\mathrm{OH} 231.8+4.2$ ), on the other hand, can drive bipolar outflows with speeds up to several hundred $\mathrm{km} \mathrm{s}^{-1}$, but as previously mentioned the duration of this phase is very short for objects more luminous than $\sim 10^{4} L_{\odot}$, and thus is rarely seen.

The well-studied DR21(OH) massive star-forming region makes a useful point for comparison as it shares several similarities with IRAS $19312+1950$, with a similar bolometric luminosity, molecular emission line spectrum (including total $\mathrm{CO}$ and $\mathrm{H}_{2} \mathrm{O}$ line luminosities), and far-IR SED (Karska et al. 2014; Jakob et al. 2007). DR21(OH) also exhibits Class I $\mathrm{CH}_{3} \mathrm{OH}$ masers and a tentative $\mathrm{SiO}$ maser detection (Kalenskii \& Johansson 2010), but without a strong $1612 \mathrm{MHz} \mathrm{OH}$ maser. Similar to IRAS $19312+1950$, the lack of $\mathrm{H} \alpha$ emission in DR21(OH) (Kumar et al. 2007) is consistent with a young evolutionary stage (and low effective temperature), implying that a large $\mathrm{H}$ II region has not yet developed.

Orion KL Source $I$ is a nearby massive protostar that possesses $\mathrm{SiO}, \mathrm{H}_{2} \mathrm{O}$, and $\mathrm{OH}(1612$ and $1665 \mathrm{MHz})$ masers, as well as a warm disk and bipolar outflow (Greenhill et al. 2004; Cohen et al. 2006; Plambeck et al. 2009). The apparent similarity of these characteristics with IRAS $19312+1950$ suggests a similar evolutionary stage. By this analogy, the origin of the $\mathrm{SiO}$ and $\mathrm{OH} 1612 \mathrm{MHz}$ masers in IRAS 19312 +1950 may also be in the (wide-angle) bipolar outflow (e.g., Cohen et al. 2006). Further studies of the structure of IRAS $19312+1950$ could be helpful to improve our understanding of the kinematically complex region surrounding Source $I$.

As highlighted by Nakashima et al. (2016), the environment surrounding IRAS $19312+1950$ appears to show a lack of prior star formation. The absence of other confirmed protostars nearby could indicate that this object is a relatively isolated site of high-mass star formation, but more detailed IR imaging studies are required to properly determine the young stellar population in this region.

\section{CONCLUDING REMARKS}

The observational evidence presented in this article leads to a self-consistent view of IRAS $19312+1950$ as a massive YSO embedded in a collapsing molecular envelope. Far-infrared SED modeling and comparison of the IR spectral features with other sources provides strong evidence to support this scenario. Indeed, the spectrum of PACS and HIFI emission lines is very similar to that observed previously in other massive protostars.
The fact that $\mathrm{SiO}$ and $\mathrm{OH}$ maser observations of IRAS 19312 +1950 have previously been considered to be more characteristic of an evolved star highlights the unusual nature of this object, making it an ideal candidate for followup observations (using ALMA, SOFIA, and JWST, for example) to confirm its identity and search for any other peculiarities that may help inform our understanding of the process of high-mass starformation/stellar evolution.

Future studies to confirm the nature of IRAS $19312+1950$ will require high-resolution imaging to elucidate the spatial and kinematic structure of the outflow, envelope, and putative disk. Observations of optically thin, dense molecular gas tracers such as $\mathrm{C}^{18} \mathrm{O}, \mathrm{CS}, \mathrm{HCN}$, and $\mathrm{H}_{2} \mathrm{CO}$ using sub-millimeter interferometry (at sub-arcsecond resolutions) should be particularly revealing. Detection and mapping of outflow tracers such as $\mathrm{CO}, \mathrm{SiO}$, and $\mathrm{HCO}^{+}$, and "hot core" chemical tracers such as $\mathrm{CH}_{3} \mathrm{CN}, \mathrm{CH}_{3} \mathrm{OCHO}$, and $\mathrm{C}_{2} \mathrm{H}_{5} \mathrm{CN}$ would also help to confirm the YSO identification. The presence of a compact $\mathrm{H}$ II region may be revealed by searching for emission from hot, ionized gas, either through deep radio continuum observations or far-IR line searches for C II, N II, and other ions.

Although our data strongly indicate the presence of a massive YSO, a chance coincidence with a massive evolved star along the line of sight still cannot be ruled out. The presence of an AGB star could be established, for example, by measuring the profile of the $3 \mu \mathrm{m} \mathrm{H}_{2} \mathrm{O}$ absorption band to determine the presence of crystalline $\mathrm{H}_{2} \mathrm{O}$. More detailed mapping of far-IR emission from dust, $\mathrm{O}$ I, and $\mathrm{H}_{2} \mathrm{O}$ would also be worthwhile to elucidate the energetic environment close to the star.

Support for this work was provided by NASA through an award issued by JPL/Caltech and through NASA's Origins of Solar Systems program. We gratefully acknowledge the work of Thomas Robitaille for providing and supporting the Hyperion radiative transfer code.

Facilities: Herschel Space Observatory, Spitzer Space Telescope.

\section{REFERENCES}

Adams, F. C., Lada, C. J., \& Shu, F. H. 1987, ApJ, 312, 788

Boogert, A. C. A., Gerakines, P. A., \& Whittet, D. C. B. 2015, ARA\&A, 53,541

Boogert, A. C. A., Pontoppidan, K. M., Knez, C., et al. 2008, ApJ, 678, 985 Boogert, A. C. A., Huard, T. L., Cook, A. M., et al. 2011, ApJ, 729, 92

Bruderer, S., Benz, A. O., Doty, S. D., van Dishoeck, E. F., \& Bourke, T. L. 2009, ApJ, 700, 872

Carpenter, J. M., Snell, R. L., \& Schloerb, F. P. 1990, ApJ, 362, 147

Casali, M., Adamson, A., Alves de Oliveira, C., et al. 2007, A\&A, 467, 777

Caswell, J. L. 1998, MNRAS, 297, 215

Churchwell, E., Walmsley, C. M., \& Cesaroni, R. 1990, A\&AS, 83, 119

Cohen, R. J., Gasiprong, N., Meaburn, J., \& Graham, M. F. 2006, MNRAS, 367,541

Cummins, S. E., Linke, R. A., \& Thaddeus, P. 1986, ApJS, 60, 819

Cyganowski, C. J., Brogan, C. L., Hunter, T. R., \& Churchwell, E. 2009, ApJ, 702,1615

Danilovich, T., Bergman, P., Justtanont, K., et al. 2014, A\&A, 569, A76 David, P., Le Squeren, A. M., \& Sivagnanam, P. 1993, A\&A, 277, 453 De Beck, E., Lombaert, R., Agundez, M., et al. 2012, A\&A, 539, A108 de Graauw, T., Helmich, F. P., \& Phillips, T. G. 2010, A\&A, 518, L6 Decin, L., Cernicharo, J., Barlow, M. J., et al. 2010, A\&A, 518, L143 Deguchi, S., Nakashima, J.-I., \& Takano, S. 2004, PASJ, 56, 1083

Dunham, M. K., Rosolowsky, E., Evans, N. J., II, Cyganowski, C., \& Urquhart, J. S. 2011, ApJ, 741, 110

Edris, K. A., Fuller, G. A., \& Cohen, R. J. 2007, A\&A, 465, 865

Elia, D., Schisano, E., Molinari, S., et al. 2010, A\&A, 518, L97 
Fish, V. L., Zschaechner, L. K., Sjouwerman, L. O., Pihlström, Y. M., \& Claussen, M. J. 2006, ApJL, 653, L45

Fuente, A., Neri, R., \& Caselli, P. 2005, A\&A, 444, 481

Gerakines, P. A., Schutte, W. A., Greenberg, J. M., \& van Dishoeck, E. F. 1995, A\&A, 296, 810

Gerakines, P. A., Schutte, W. A., Greenberg, J. M., \& van Dishoeck, E. F. 1999, ApJ, 522, 357

Ginsburg, A., Walsh, A., Henkel, C., et al. 2015, A\&A, 584, L7

Goicoechea, J. R., Cernicharo, J., Karska, A., et al. 2012, A\&A, 548, A77

Grave, J. M. C., \& Kumar, M. S. N. 2009, A\&A, 498, 147

Greenhill, L. J., Reid, M. J., Chandler, C. J., Diamond, P. J., \& Elitzur, M 2004, in IAU Symp. 221, Star Formation at High Angular Resolution, ed. M. G. Burton, R. Jayawardhana, \& T. L. Bourke (San Francisco, CA: ASP), 155

Groenewegen, M. A. T., Waelkens, C., Barlow, M. J., et al. 2011, A\&A, 526, A162

Harwit, M., Malfait, K., Decin, L., et al. 2001, ApJ, 557, 844

Herman, J., \& Habing, H. J. 1985, A\&AS, 59, 523

Hernández, J., Calvet, N., Briceño, C., Hartmann, L., \& Berlind, P. 2004, AJ, 127,1682

Hewett, P. C., Warren, S. J., Leggett, S. K., \& Hodgkin, S. T. 2006, MNRAS, 367,454

Hodgkin, S. T., Irwin, M. J., Hewett, P. C., \& Warren, S. J. 2009, MNRAS, 394, 675

Hollenbach, D. J., \& Tielens, A. G. G. M. 1997, ARA\&A, 35, 179

Hunter, T. R., Young, K. H., Christensen, R. D., \& Gurwell, M. A. 2007, in IAU Symp. 242, Astrophysical Masers and their Environments, ed J. M. Chapman \& W. A. Baan (Cambridge: Cambridge Univ. Press), 481

Imai, H., Tafoya, D., Honma, M., Hirota, T., \& Miyaji, T. 2011, PASJ, 63, 81

Jakob, H., Kramer, C., Simon, R., et al. 2007, A\&A, 461, 999

Justtanont, K., Barlow, M. J., Tielens, A. G. G. M., et al. 2000, A\&A, 360, 1117

Kalenskii, S. V., \& Johansson, L. E. B. 2010, ARep, 54, 295

Karska, A., Herpin, F., Bruderer, S., et al. 2014, A\&A, 562, A45

Kastner, J. H., \& Myers, P. C. 1994, ApJ, 421, 605

Keto, E., Caselli, P., \& Rawlings, J. 2015, MNRAS, 446, 3731

Khouri, T., de Koter, A., Decin, L., et al. 2014, A\&A, 561, A5

Kristensen, L. E., van Dishoeck, E. F., Bergin, E. A., et al. 2010, A\&A, 521, L30

Kristensen, L. E., Visser, R., van Dishoeck, E. F., et al. 2012, A\&A, 542, A8

Kumar, M. S. N., Davis, C. J., Grave, J. M. C., Ferreira, B., \& Froebrich, D. 2007, MNRAS, 374, 54

Lada, C. J. 1987, in IAU Symp. 115, Star Forming Regions, ed. M. Peimbert \& J. Jugaku (Dordrecht: Reidel), 1

Lawrence, A., Warren, S. J., Almaini, O., et al. 2007, MNRAS, 379, 1599

Lee, S., Lee, J.-E., \& Bergin, E. A. 2015, ApJS, 217, 30

Liseau, R., Justtanont, K., \& Tielens, A. G. G. M. 2006, A\&A, 446, 561

Manoj, P., Watson, D. M., Neufeld, D. A., et al. 2013, ApJ, 763, 83

Markwardt, C. 2012, Robust nonlinear least squares curve fitting Astrophysics Source Code Library, ascl:1208.019

Mauron, N., \& Josselin, E. 2011, A\&A, 526, A156
McKee, C. F., \& Ostriker, E. C. 2007, ARA\&A, 45, 565

Molinari, S., Pezzuto, S., Cesaroni, R., et al. 2008, A\&A, 481, 345

Molinari, S., Swinyard, B., Bally, J., et al. 2010, A\&A, 518, L100

Mottram, J. C., Kristensen, L. E., van Dishoeck, E. F., et al. 2014, A\&A, 572, A21

Murakawa, K., Nakashima, J., Ohnaka, K., \& Deguchi, S. 2007, A\&A, 470,957

Nakashima, J.-i., Ladeyschikov, D. A., Sobolev, A. M., et al. 2016, ApJ, 825,16

Nakashima, J.-i., \& Deguchi, S. 2000, PASJ, 52, L43

Nakashima, J.-i., \& Deguchi, S. 2005, ApJ, 633, 282

Nakashima, J.-i., Deguchi, S., Imai, H., Kemball, A., \& Lewis, B. M. 2011 ApJ, 728, 76

Nakashima, J.-i., Sobolev, A. M., Salii, S. V., et al. 2015, PASJ, 67, 95

Ott, S. 2010, in ASP Conf. Ser. 434, Astronomical Data Analysis Software and Systems XIX, ed. Y. Mizumoto, K.-I. Morita, \& M. Ohishi, (San Francisco, CA: ASP), 139

Pilbratt, G. L., Riedinger, J. R., Passvogel, T., et al. 2010, A\&A, 518, L1

Plambeck, R. L., Wright, M. C. H., Friedel, D. N., et al. 2009, ApJL, 704, L25

Poglitsch, A., Waelkens, C., Geis, N., et al. 2010, A\&A, 518, L2

Povich, M. S., Churchwell, E., Bieging, J. H., et al. 2009, ApJ, 696, 1278

Purcell, C. R., Hoare, M. G., Cotton, W. D., et al. 2013, ApJS, 205, 1

Ramachandran, R., Deshpande, A. A., \& Goss, W. M. 2006, ApJ, 653, 1314

Robitaille, T. P., et al. 2011, A\&A, 536, A79

Robitaille, T. P., Whitney, B. A., Indebetouw, R., Wood, K., \& Denzmore, P. 2006, ApJS, 167, 256

Robitaille, T. P., Meade, M. R., Babler, B. L., et al. 2008, AJ, 136, 2413

Rosolowsky, E., Dunham, M. K., Ginsburg, A., et al. 2010, ApJS, 188, 123

Roussel, H. 2013, PASP, 125, 1126

Royer, P., Decin, L., Wesson, R., et al. 2010, A\&A, 518, L145

Rygl, K. L. J., Wyrowski, F., Schuller, F., \& Menten, K. M. 2013, A\&A, 549, A5

San José-García, I., Mottram, J. C., van Dishoeck, E. F., et al. 2016, A\&A, 585, A 103

Scott, K. 2002, BAAS, 34, 1216

Sylvester, R. J., Kemper, F., Barlow, M. J., et al. 1999, A\&A, 352, 587

te Lintel Hekkert, P., \& Chapman, J. M. 1996, A\&AS, 119, 459

Tobin, J. J., Stutz, A. M., Megeath, S. T., et al. 2015, ApJ, 798, 128

Torrelles, J. M., Gómez, J. F., Rodríguez, L. F., et al. 1998, ApJ, 505, 756

Traficante, A., Calzoletti, L., Veneziani, M., et al. 2011, MNRAS, 416, 2932

Ulrich, R. K. 1976, ApJ, 210, 377

van Kempen, T. A., Kristensen, L. E., Herczeg, G. J., et al. 2010, A\&A, 518, L121

van Loon, J. T., Oliveira, J. M., Gordon, K. D., et al. 2010, AJ, 139, 68

van Winckel, H. 2003, ARA\&A, 41, 391

Visser, R., Kristensen, L. E., Bruderer, S., et al. 2012, A\&A, 537, A55

Wampfler, S. F., Herczeg, G. J., Bruderer, S., et al. 2010, A\&A, 521, L36

Whitney, B. A., Wood, K., Bjorkman, J. E., \& Wolff, M. J. 2003, ApJ, 591, 1049

Yoon, D.-H., Cho, S.-H., Kim, J., Yun, Y. j., \& Park, Y.-S. 2014, ApJS, 211,15

Zapata, L. A., Menten, K., Reid, M., \& Beuther, H. 2009, ApJ, 691, 332 